\title{
Association of expression of epigenetic molecular factors with DNA methylation and sensitivity to chemotherapeutic agents in cancer cell lines
}

Suleyman Vural', Alida Palmisano 1,20, William C. Reinhold ${ }^{3}$, Yves Pommier $^{3}$, Beverly A. Teicher $^{4}$ and Julia Krushkal ${ }^{1 *}$ (1)

\begin{abstract}
Background: Altered DNA methylation patterns play important roles in cancer development and progression. We examined whether expression levels of genes directly or indirectly involved in DNA methylation and demethylation may be associated with response of cancer cell lines to chemotherapy treatment with a variety of antitumor agents.

Results: We analyzed 72 genes encoding epigenetic factors directly or indirectly involved in DNA methylation and demethylation processes. We examined association of their pretreatment expression levels with methylation betavalues of individual DNA methylation probes, DNA methylation averaged within gene regions, and average epigenome-wide methylation levels. We analyzed data from 645 cancer cell lines and 23 cancer types from the Cancer Cell Line Encyclopedia and Genomics of Drug Sensitivity in Cancer datasets. We observed numerous correlations between expression of genes encoding epigenetic factors and response to chemotherapeutic agents. Expression of genes encoding a variety of epigenetic factors, including KDM2B, DNMT1, EHMT2, SETDB1, EZH2, APOBEC3G, and other genes, was correlated with response to multiple agents. DNA methylation of numerous target probes and gene regions was associated with expression of multiple genes encoding epigenetic factors, underscoring complex regulation of epigenome methylation by multiple intersecting molecular pathways. The genes whose expression was associated with methylation of multiple epigenome targets encode DNA methyltransferases, TET DNA methylcytosine dioxygenases, the methylated DNA-binding protein ZBTB38, KDM2B, SETDB1, and other molecular factors which are involved in diverse epigenetic processes affecting DNA methylation. While baseline DNA methylation of numerous epigenome targets was correlated with cell line response to antitumor agents, the complex relationships between the overlapping effects of each epigenetic factor on methylation of specific targets and the importance of such influences in tumor response to individual agents require further investigation.

Conclusions: Expression of multiple genes encoding epigenetic factors is associated with drug response and with DNA methylation of numerous epigenome targets that may affect response to therapeutic agents. Our findings suggest complex and interconnected pathways regulating DNA methylation in the epigenome, which may both directly and indirectly affect response to chemotherapy.
\end{abstract}

\footnotetext{
*Correspondence: julia.krushkal@nih.gov

${ }^{1}$ Biometric Research Program, Division of Cancer Treatment

and Diagnosis, National Cancer Institute, 9609 Medical Center Dr.,

Rockville, MD 20850, USA

Full list of author information is available at the end of the article
} permits use, sharing, adaptation, distribution and reproduction in any medium or format, as long as you give appropriate credit to the original author(s) and the source, provide a link to the Creative Commons licence, and indicate if changes were made. The images or other third party material in this article are included in the article's Creative Commons licence, unless indicated otherwise in a credit line to the material. If material is not included in the article's Creative Commons licence and your intended use is not permitted by statutory regulation or exceeds the permitted use, you will need to obtain permission directly from the copyright holder. To view a copy of this licence, visit http://creativecommons.org/licenses/by/4.0/. The Creative Commons Public Domain Dedication waiver (http://creativeco mmons.org/publicdomain/zero/1.0/) applies to the data made available in this article, unless otherwise stated in a credit line to the data. 
Keywords: DNA methylation, Gene expression, Epigenetic analysis, Cancer drug treatment

\section{Background}

Cancer cells acquire multiple epigenomic alterations, including aberrant DNA methylation and DNA hydroxymethylation of genes and genome regions, loss or gain of imprinting and allele switching of imprinted loci, and global DNA hypomethylation [1-6]. Epigenetic changes in malignant cells result in transcriptional and posttranscriptional rewiring, influencing cell cycle, growth, and proliferation. Epigenetic dysregulation in tumors leads to silencing of tumor suppressor genes and of genes involved in DNA repair, activates oncogene expression, alters gene function, affects transcriptional regulatory networks, and increases genome instability $[1,4$, 6-11]. Global DNA hypomethylation of malignant cells has been associated with tumor evasion of the immune response [12].

Many epigenetic factors directly or indirectly dynamically influence genome region-specific or global DNA methylation in the germ line, embryonic, or somatic adult cells (Additional file 1: Table S1). Below, we refer to their genes as GMDs (genes affecting DNA methylation or demethylation). Products of the DNA methyltransferase (DNA $5^{\prime}$ cytosine-methyltransferase, or DNMT) genes DNMT1, DNMT3A and DNMT3B are directly involved in DNA methylation. Products of TET methylcytosine dioxygenase genes (TET1, TET2 and TET3) and products of AICDA (AID) and APOBEC, TDG, MBD4, $S M U G 1$, and GADD45A participate in DNA demethylation through DNA hydroxymethylation, deamination, base excision repair (BER), and other mechanisms [4, 1319]. For example, a molecular complex containing AID, TDG, and GADD45A participates in DNA demethylation via the BER pathway [20].

Many factors participate in molecular complexes that affect DNA methylation or demethylation, participate in methylation-dependent targeting of other molecular factors to genome regions, or regulate binding and/or activities of DNMTs, TETs, and other epigenetic factors, either directly or via intermediate metabolites. Examples include MBD1, MBD2, MBD3, MBD4, PCNA, USP7 (HAUSP), DNMT3L, UHRF1, UHRF2, DMAP1, ZBTB4, ZBTB33 (KAISO), ZBTB38, RBPJ, G9A (EHMT2), KAT5 (TIP60), SUV39H1, HDAC1, SIRT1, EZH2, CSNK1D, CSNK1E, and SUMO1 (Additional file 1: Table S1) [4, 13, 21-29]. IDH1 and IDH2 mutations lead to overproduction of cellular metabolites which interfere with TETmediated conversion of 5-methylcytosine $(5-\mathrm{mC})$ to 5-hydroxymethylcytosine (5-hmC) [30]. GLP (EHMT1), G9A (EHMT2), ZFP57, DPPA3 (PGC7, or STELLA),
TRIM28 (KAP1), SETDB1, DNMT3L, EED, EZH2, SUZ12, and ZSCAN4 are involved in regulation, de novo methylation, and/or maintenance of imprinted regions and/or affect DNA methylation in embryonic stem cells [18, 27, 31-36].

While many GMDs are involved in methylation or demethylation of 5-mC, MGMT demethylates $\mathrm{O}^{6}$-methylguanine $\left(\mathrm{O}^{6}\right.$-meG) lesions and removes $\mathrm{O}^{6}$-alkyl adducts, whereas ALKBH2 and ALKBH3 demethylate DNA via removal of 1-methyladenine $\left(\mathrm{N}^{1}-\mathrm{meA}\right)$ and 3-methylcytosine $\left(\mathrm{N}^{3}\right.$-meC) [37-39].

A number of epigenetic factors have complex and intertwined roles affecting DNA methylation. There is an extensive cross-talk among the DNA methylation, demethylation, and histone modification pathways in germ line, embryonic stem, normal somatic, and malignant cells $[26,27,31,35,40-42]$. DNA methylation is influenced by histone modifications, and histone methylation and acetylation marks directly affect DNMT localization, binding, and activities [27, 35, 40, 43]. Specific GMD roles in DNA methylation and demethylation and examples of their interactions are presented in Additional file 1: Table S1 and accompanying text.

GMD components may directly or indirectly affect sensitivity of cancer cells to treatment. DNMTs are directly inhibited by DNA hypomethylating agents, while other antitumor agents target additional GMD products [1, 7, 44-49]. The Hsp90 inhibitor 17-DMAG diminishes the binding of DNMT1 and of the histone methyltransferase EZH2 to Hsp90, attenuates the interaction between DNMT1 and EZH2, and mediates the depletion of DNMT1 and EZH2 [50]. HDAC inhibitors (HDACi) affect DNA methylation through a variety of mechanisms. Vorinostat downregulates transcription of $D N M T 1$ and DNMT3B and changes DNA methylation of TERT and DLC1 [51-53]. Panobinostat depletes protein levels of DNMT1 and EZH2 and disrupts DNMT1 interaction with $\mathrm{EZH} 2$ and the polycomb repressive complex 2 (PRC2) [50]. Trichostatin A downregulates gene and protein expression of DNMT1 and induces global DNA hypomethylation [54]. Belinostat reduces global DNA methylation and depletes protein levels of the PRC2 subunits EZH2 and SUZ12 [55].

Among examples of the influence of DNA methylation on tumor sensitivity to treatment, MGMT promoter methylation downregulates $M G M T$ expression, disrupting MGMT role in DNA repair, which is linked to resistance to nitrosourea-based antitumor agents, temozolomide, and radiation [37, 56, 57]. Specific DNA 
methylation patterns or methylation of individual genes have been associated with resistance to different types of cancer drugs, e.g., the platinum compound cisplatin, poly(ADP-ribose) polymerase (PARP) inhibitors, the microtubule-disrupting agent paclitaxel, and the cytidine analog cytarabine [11, 57-66], which may suggest indirect influences of GMD on drug response.

Due to the significance of epigenetic factors in regulation of DNA methylation, it is important to investigate how GMD expression may directly or indirectly affect tumor response to treatment (Fig. 1). We used cancer cell line data from two public resources, the Cancer Cell Line Encyclopedia (CCLE) and the Genomics of Drug Sensitivity in Cancer [67-72], to examine associations of drug response with 72 GMDs (Additional file 1: Table S1) that are directly or indirectly involved in DNA methylation or demethylation. We investigated correlations of their pretreatment expression with methylation of their putative genome targets and with cancer cell line response to a variety of antitumor agents with different mechanisms of action.

\section{Methods}

Selection of candidate genes involved in DNA methylation and demethylation

Additional file 1: Table S1 provides the list of the 72 GMDs analyzed in this study. Their products are directly or indirectly involved in DNA methylation or demethylation in human tissues. Information about their biological roles in DNA methylation or demethylation was obtained from the biomedical literature and from GeneCards [73] and the Online Mendelian Inheritance in Men (OMIM) [74].

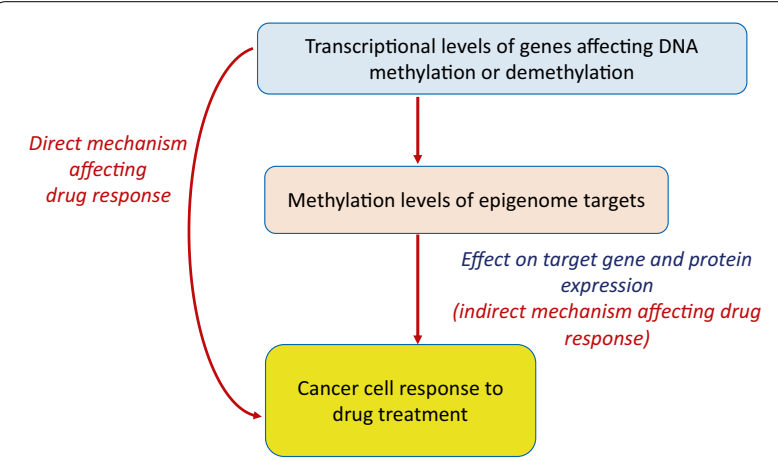

Fig. 1 Possible hypothetical mechanisms by which GMD expression may directly or indirectly affect response of cancer cells drugs. GMDs may directly influence drug response through a variety of mechanisms. Among indirect influences of GMDs on drug response examined in this study, we focused on the effect of GMD expression on DNA methylation of epigenome targets

\section{Drug response data}

To examine the relationship between pretreatment GMD expression and tumor response to antitumor agents, we used gene expression, DNA methylation, and drug response data for 645 cell lines, the identity of which was matched between the Cancer Cell Line Encyclopedia and the Genomics of Drug Sensitivity in Cancer datasets [67-72] (Additional file 2: Table S2). The IC50 measures of drug response, representing the total drug concentration that reduced cell activity by $50 \%$, were available for 24 agents from CCLE [67, 68, 72]. Additional IC50 values for 251 agents were obtained from the Genomics of Drug Sensitivity in Cancer portal $[69,71,75]$. Below were refer to these drug response measures as GDSC measures. After our analysis was completed, GDSC released a second batch of drug response values, referring in their release to the initial dataset as GDSC1 and the second dataset as GDSC2 [75]. All GDSC data analyzed in our study were from the GDSC1 dataset.

All CCLE and GDSC drug sensitivity values were transformed to the $\log _{10}$ (IC50) scale. Cell line identities in the CCLE and GDSC datasets were verified using Cellosaurus [76]. Response measures for 11 agents which were present in both CCLE and GDSC data were analyzed separately, without combining the CCLE and GDSC measures. For those agents in the GDSC dataset that had duplicate measurements [71], we used the combined average of their drug response measures from separate experiments. The resulting dataset included 275 CCLE and GDSC drug response measures for 255 distinct antitumor agents. The concordance of drug response measures between the CCLE and GDSC datasets has been reported previously [77-79]. Information about mechanisms of action of the agents was collected from the CCLE and GDSC portals, their accompanying publications $[67,68,71,75]$ and biomedical literature.

\section{Gene expression data retrieval}

For the RNA-seq data used in this project, RPKM gene expression values were downloaded from the CCLE portal of the Broad Institute [72, 80]. RNA sample library preparation using Illumina TruSeq RNA Sample Preparation protocol, RNA-sequencing using Illumina HiSeq 2000 and HiSeq 2500, and initial data processing was previously described by the CCLE project [81].

\section{DNA methylation data filtering}

Cell line methylation data for 485,512 probes, generated by the GDSC project [71] using Illumina Infinium HumanMethylation450 (450 K) BeadChip array (Illumina, Inc.), were downloaded from NCBI GEO [82]. Methylation probe beta-values for individual cell lines 
with detection $p$-values $\geq 10^{-3}$ and 340 entire probes with median detection $p$-values $\geq 10^{-6}$ were excluded. In addition, 60,332 probes overlapping with single nucleotide polymorphisms were filtered out based on the probe masking recommendations for hg19 (GRCh37) [83, 84]. The final methylation dataset used in analysis had methylation beta-values for 424,840 probes that passed all filtering. Chromosomal regions (cytobands) were identified according to the UCSC genome annotation for the hg19 (GRCh37) human genome assembly based on the probe coordinates in the Illumina Infinium HumanMethylation $450 \mathrm{~K}$ BeadChip annotation.

\section{Calculation of gene region-averaged methylation values}

In order to compute gene region-averaged methylation beta-values from individual probe measures, we developed an $\mathrm{R}$ program (available upon request) which followed the algorithm developed previously by the authors of the IMA software [85]. We recently reported a version of our software adapted for the Illumina Infinium MethylationEPIC BeadChip array [86]. For this study, we used a similar version which we adapted for the Illumina Infinium HumanMethylation450 BeadChip array. We used the Illumina Infinium HumanMethylation450 BeadChip annotation of each probe [87] according to the UCSC genome browser data to compute gene regionaveraged methylation for 6 gene regions: TSS1500 (2001500 bases upstream of the transcriptional start site, or TSS), TSS200 (0-200 bases upstream of the TSS), 5'UTR (within the $5^{\prime}$ untranslated region, between the TSS and the ATG start site), 1st exon, gene body (between the ATG start site and the stop codon), and 3'UTR (within the $3^{\prime}$ untranslated region, between the stop codon and poly A signal). The resulting methylation values were computed for 93,591 regions in 20,643 genes and ncRNA, with each gene represented by up to 6 regions. Additional file 20: Fig. S1 shows the distribution of methylation betavalues among 424,840 individual probes, the combined distribution among 93,591 gene regions, and separate distributions for each gene region category (TSS1500, TSS200, 5'UTR, 1st exon, gene body, and 3' UTR) in 645 cell lines.

\section{Association analysis of GMD expression, epigenome-wide methylation of individual probes and gene regions, and drug response}

To examine possible direct influences of GMD expression on drug response (Fig. 1), we analyzed Spearman correlation between RPKM expression measures of 72 GMDs listed in Additional file 1: Table S1 and $\log (\mathrm{IC} 50)$ of 255 antitumor agents. Significance of the associations was evaluated using the Benjamini-Hochberg adjustment procedure for false discovery rate (FDR) [17], while accounting for 255 agents and 72 genes. We identified the associations between GMD expression and $\log$ (IC50) which were both statistically significant (satisfying FDR adjusted $p<0.05$ ) and strong (satisfying the absolute value of Spearman correlation coefficient $|\rho|>0.5$ ) (Fig. 2a). Here and below, we refer to the FDR adjusted $p$-values as $p_{\mathrm{FDR}}$. We discuss the strength of statistically significant associations based on the absolute value of their Spearman correlation coefficient $|\rho|$.

All association analyses were performed in the combined dataset of different cancer categories (pancancer analysis including 645 cell lines), and also separately within each of the 23 individual cancer categories with at least 10 cell lines that had both methylation and expression data (Additional file 2: Table S2). The initial information about tumor sites was obtained from GDSC, CCLE, and Cellosaurus [67-69, 71, 72, 75, 76]. While many cancer categories used in our analysis were based on the Cancer Genome Atlas (TCGA) definitions, some cancer types from the same organ were grouped into broader categories in order to allow for an inclusion of a broader range of similar cell lines than those defined by TCGA. Several additional categories not presented in TCGA (e.g., small cell lung cancer, neuroblastoma, and others) were also analyzed (Additional file 2: Table S2). In the analysis stratified by individual cancer categories with $\geq 10$ cell lines, we accounted for 23 cancer types in the FDR adjustment.

We also explored potential indirect mechanisms which may mediate the associations between GMD expression and drug response. We used Spearman correlation to identify the strongest significant associations between expression of the 72 GMDs and methylation of their epigenome targets by using methylation beta-values of 424,840 individual probes and 93,591 gene region methylation values averaged among the probes within each region (Fig. 2b). Among individual probes, in the combined pancancer analysis of all tumor types we searched for associations between GMD expression and methylation beta values with $p<\frac{10^{-6}}{72}$, i.e., $p<1.389 \times 10^{-8}$, based on published recommendations [88] for the $p$-value threshold that would be appropriate for finding single gene associations with methylation probes of the HumanMethylation $450 \mathrm{~K}$ BeadChip array. We adjusted it by the number of GMDs for which the associations were examined. When analyzing associations between GMD expression and individual probes within each of the 23 cancer categories, we further adjusted this threshold by using $p<\frac{1.38910^{-8}}{23}=6.039 \times 10^{-10}$. In the pancancer correlation analysis between GMD expression and gene region methylation, we used the FDR adjustment that accounted for 72 GMDs and 93,591 gene regions. In correlation analyses between GMD expression and gene 
a

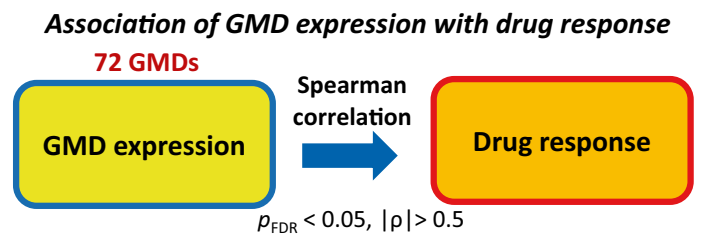

b

Association of GMD expression with epigenome-wide methylation of probes and gene regions to identify significant targets

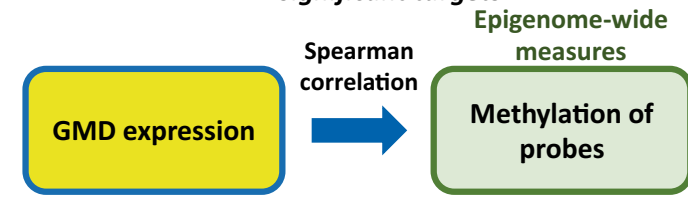

Pancancer analysis: $p<1.38910^{-8},|\rho|>0.5$

Analysis of individual cancer categories: $p<6.03910^{-10},|\rho|>0.5$

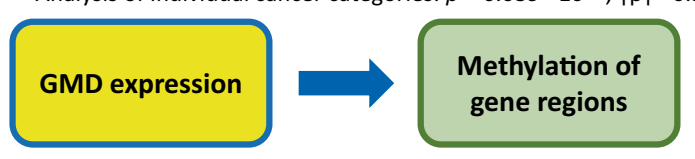

$p_{\mathrm{FDR}}<0.05,|\rho|>0.5$

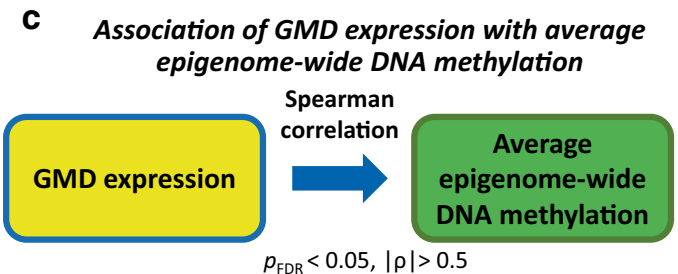

Association of drug response with methylation of target probes and gene regions which were significantly associated with GMD expression Epigenome targets associated with GMDs

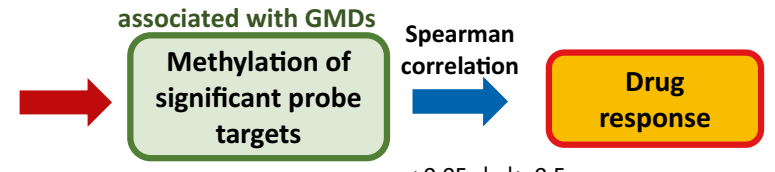
$p_{\mathrm{FDR}}<0.05,|\rho|>0.5$

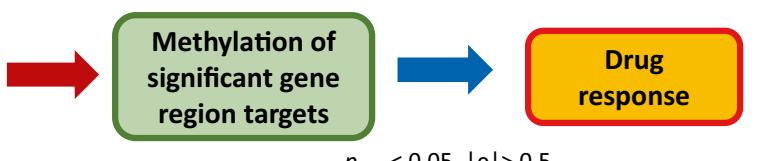

$p_{\mathrm{FDR}}<0.05,|\rho|>0.5$

\section{Association of average epigenome-wide DNA methylation with drug response}

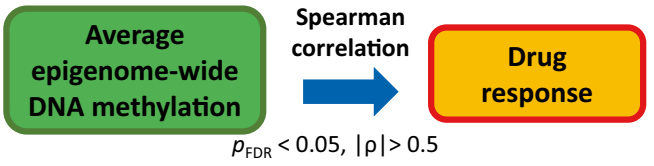

Fig. 2 Overall design of the study. a Analysis of direct associations between GMD expression and drug response. $\mathbf{b}$ A two-step approach to identify possible indirect GMD effects on drug response. Epigenetic targets significantly associated with GMD expression were identified first, and then, the correlation of methylation of these significant targets with drug response was analyzed. c. Analysis of average DNA methylation values. In each analysis, we examined associations in all cancer categories combined (pancancer analysis) and in individual cancer types with $\geq 10$ cell lines. GMD expression data included RNA-seq RPKM values for 72 GMDs. Epigenome-wide DNA methylation data included beta-values for 424,840 individual probes and gene region-averaged values for 93,591gene regions from 20,643 genes and noncoding RNA. Average epigenome-wide methylation values were computed as a mean of beta-values for 424,840 probes which passed the QC and filtering. Drug response measures consisted of 275 $\log ($ IC50) values for 255 anticancer agents obtained from CCLE and GDSC datasets. The criteria for identifying significant strong associations are provided below each diagram. $p_{\mathrm{FDR} \text { ' }} p$-value adjusted for false discovery rate according to Benjamini-Hochberg procedure

region methylation stratified by cancer types, we also accounted for the 23 cancer types. In addition to using the $p$-value threshold, we focused on the strongest correlations that had the absolute value of Spearman $|\rho|>0.5$. We made a distinction between the cis-correlations of expression of a GMD with methylation of its own probes (which could suggest the regulation of expression of that GMD by its methylation, or a possible copy number variation of that GMD which may affect both its methylation and expression measures) and the trans-correlations of each GMD with the probes located in other genes, according to the UCSC annotation of the HumanMethylation $450 \mathrm{~K}$ BeadChip array.

After identifying putative epigenome targets that were strongly and significantly correlated with GMD expression (Fig. 2b), we examined associations of DNA methylation of these epigenome targets with drug response. Spearman correlation analysis of methylation measures with $\log (\mathrm{IC} 50)$ of each of the 255 agents was performed for methylation beta-values of the target methylation probes and gene regions that had been strongly and significantly associated with GMD expression. Significance of correlation of methylation of individual target probes or gene regions with GMD expression and with $\log (\mathrm{IC} 50)$ was evaluated using the FDR adjustment, accounting for 255 agents and the number of target methylation probes and gene regions. Analysis within cancer categories also accounted for 23 cancer types. We focused on the strongest significant correlations with $|\rho|$ $>0.5$. If the number of such correlations was small, we 
provided an additional discussion of more modest significant correlations with $|\rho|>0.4$.

In addition to the analysis of individual probes and gene regions, we also examined the association of GMD expression with epigenome-averaged DNA methylation and of epigenome-averaged DNA methylation with $\log (\mathrm{IC} 50)$ of antitumor agents (Fig. 2c). Epigenome-wide averaged DNA methylation was computed as a mean of beta-values among 424,840 methylation probes which passed the quality control (QC) and probe filtering. The resulting $p$-values were FDR adjusted for multiple testing. Separate analyses of average epigenome-wide DNA methylation were performed in the pancancer data and within 23 individual cancer categories with $\geq 10$ cell lines.

Analyses were performed using Python v. 2.7.15, R v. 3.5.3, and rpy2 v. 2.8.5.

\section{Regression analysis of associations of cell line response to trametinib}

Among the agents which were associated with GMD expression or with methylation status of epigenome targets in our study, sensitivity and resistance to the MEK inhibitor trametinib have been previously associated with specific DNA and protein sequence changes including BRAF V600E and KRAS or NRAS protein-changing variants [89, 90]. For those GMDs and target gene regions and probes which were significantly and strongly $(|\rho|$ $>0.5$ ) associated with response to trametinib either in the pancancer dataset or in any individual cancer category, we performed a regression analysis conditional on the presence of BRAF V600E or any non-synonymous KRAS or NRAS variant as predictors of trametinib response. We examined whether the association of GMD expression or methylation of their epigenome targets with response to trametinib remained statistically significant after accounting for the gene sequence variants known to affect sensitivity or resistance to trametinib. Information about the sequence variants in BRAF, KRAS, and NRAS was obtained from GDSC whole exome sequencing data [75]. Regression analysis was performed using the Imtest $\mathrm{R}$ package v. 0.9-36 for testing linear regression models, using $\log (\mathrm{IC} 50)$ as a dependent variable, and gene mutation status and GMD expression or probe or gene region methylation as predictor variables. The $p$-values for association of response to trametinib with GMD expression or with target probe and gene region methylation were FDR adjusted for multiple testing.

\section{Validation of the top study findings in publicly available independent datasets}

In order to validate the top results from our correlation analyses between GMD expression, epigenome target methylation, and drug response, we used publicly available comprehensive independent datasets containing drug response, DNA methylation, and gene expression measures. Our first validation analysis used the NCI-60 cancer cell line panel dataset, previously screened by the National Cancer Institute, which we analyzed using CellminerCDB v. 1.2 [48, 91-93]. In the CellminerCDB analysis of NCI-60 cell line panel data, we examined Pearson correlation between GMD expression (measured as $\log 2$ of averaged gene expression measures from five microarray platforms, Affymetrix Human Genome HG-U95, Affymetrix Human Genome HG-U133, Affymetrix Human Genome U133 Plus 2.0, Affymetrix GeneChip Human Exon 1.0 ST, and Agilent Whole Human Genome Oligo arrays) and log (GI50) measures of drug response (representing drug activity measures in CellminerCDB multiplied by -1 , in order to make the correlations in the CCLE-GDSC and NCI-60 datasets directly comparable) [48, 94]. We also analyzed NCI-60 data using CellminerCDB in order to validate significant correlations of GMD expression with target DNA methylation in the pancancer data. Because CellminerCDB utilizes gene level DNA methylation values which are inferred from probes located predominantly in the upstream gene regions [95], we used CellminerCDB NCI-60 DNA methylation data to confirm significant CCLE-GDSC associations of DNA methylation of upstream gene regions (TSS1500, TSS200, 5'UTR, and the 1st exon). CellminerCDB employs Pearson correlation in its analyses.

The second validation analysis used the NCI SCLC cell line dataset, containing measures for 66 small cell line cancer cell lines $[86,96]$. It is available from the NCI Small Cell Lung Cancer Project site [97], with SCLC DNA methylation and transcript expression data also available from NCBI GEO (accession numbers GSE145156 and GSE73160). In the validation analysis using the NCI SCLC cell line data, we used Pearson correlation to examine associations of GMD expression, measured using Affymetrix GeneChip ${ }^{\circledR}$ Human Exon 1.0 ST Array, with $\log (\mathrm{IC} 50)$ measures of drug response, and Spearman correlation to analyze associations between DNA methylation of individual probes, measured using Illumina Infinium MethylationEPIC BeadChip, and average methylation of gene regions, with GMD expression and with $\log ($ IC50) measures of drug response, using methodology described in our earlier report [86]. Measures of miRNA methylation were not included in the validation analysis of SCLC data.

For additional validation of the epigenome targets identified in our analysis of CCLE-GDSC data, we explored the clinical relevance of the findings in pancreatic ductal adenocarcinoma (PAAD) based on the 
literature reports which analyzed patient survival data in TCGA and in other patient datasets.

\section{Searchable online resource}

Our analysis generated an extensive set of tables with detailed information about the associations of genes affecting DNA methylation or demethylation. In order to provide the scientific community with the opportunity to independently explore these associations, we developed a web resource with dynamic searching and filtering features. The web resource is available at https ://brb.nci.nih.gov/gmdtables/. It was developed using HTML, CSS, and the DataTables Javascript plug-in as highly flexible tools that allow researchers to visualize, search, filter, and download our results data for their own use. The online site also provides information about the 645 cancer cell lines used in our analysis.

\section{Results}

Association of GMD expression with drug response

Table 1 summarizes significant associations of GMD expression with $\log (\mathrm{IC} 50)$ which satisfied Spearman $|\rho|$ $>0.4$ and $p_{\mathrm{FDR}}<0.05$. Seven negative and positive correlations in individual cancer categories satisfied $p_{\mathrm{FDR}}<0.05$. All of them were strong $(0.5171 \leq|\rho| \leq 0.7900$; Table 1$)$. The highest number (4) of significant associations was observed in breast cancer.

Pancancer correlations were highly significant but did not reach $|\rho|>0.5$. Four genes had modest correlations with $|\rho|>0.4$ (Fig. 3a), all of which were negative, indicating that increased GMD expression was associated with drug sensitivity. They were $K D M 2 B$ (13 correlations), DNMT1 (3), APOBEC3G (1), and EHMT2 (1). Additional file 3: Table S3 and Fig. 3b provide an expanded list of 379 significant pancancer correlations satisfying a relaxed threshold of $|\rho|>0.3$ and $p_{\mathrm{FDR}}<0.05$. In the majority of them $(91.8 \%$, or 348 out of 379 correlations), increased GMD expression was associated with

Table 1 Significant correlations of GMD expression with drug response in the pancancer dataset and in individual cancer categories satisfying $|\rho|>0.4$ and $p_{\mathrm{FDR}}<0.05$

\begin{tabular}{|c|c|c|c|c|c|}
\hline Cancer category & GMD & Agent & $\rho$ & $p_{\mathrm{FDR}}$ & Sample size \\
\hline Pancancer & $K D M 2 B$ & XMD13-2 & -0.4319 & $3.99 \times 10^{-24}$ & 590 \\
\hline Pancancer & $K D M 2 B$ & BMS-345541 & -0.4313 & $3.99 \times 10^{-24}$ & 590 \\
\hline Pancancer & $K D M 2 B$ & T0901317 & -0.4231 & $3.83 \times 10^{-23}$ & 586 \\
\hline Pancancer & DNMT1 & Zibotentan & -0.4214 & $3.83 \times 10^{-23}$ & 591 \\
\hline Pancancer & $K D M 2 B$ & NPK76-II-72-1 & -0.4174 & $1.01 \times 10^{-22}$ & 591 \\
\hline Pancancer & APOBEC3G & Z-LLNle-CHO & -0.4148 & $2.64 \times 10^{-9}$ & 225 \\
\hline Pancancer & $K D M 2 B$ & Zibotentan & -0.4139 & $2.37 \times 10^{-22}$ & 591 \\
\hline Pancancer & $K D M 2 B$ & Quizartinib & -0.4095 & $8.98 \times 10^{-22}$ & 589 \\
\hline Pancancer & $K D M 2 B$ & UNC1215 & -0.4062 & $5.08 \times 10^{-21}$ & 574 \\
\hline Pancancer & $K D M 2 B$ & Daporinad & -0.4057 & $7.77 \times 10^{-21}$ & 569 \\
\hline Pancancer & $K D M 2 B$ & Vorinostat & -0.4057 & $2.13 \times 10^{-19}$ & 527 \\
\hline Pancancer & DNMT1 & XMD13-2 & -0.4043 & $2.87 \times 10^{-21}$ & 590 \\
\hline Pancancer & EHMT2 & NPK76-II-72-1 & -0.4041 & $2.87 \times 10^{-21}$ & 591 \\
\hline Pancancer & DNMT1 & Daporinad & -0.4036 & $1.21 \times 10^{-20}$ & 569 \\
\hline Pancancer & $K D M 2 B$ & XMD14-99 & -0.4035 & $3.24 \times 10^{-21}$ & 590 \\
\hline Pancancer & $K D M 2 B$ & BX-912 & -0.4031 & $3.37 \times 10^{-21}$ & 590 \\
\hline Pancancer & $K D M 2 B$ & I-BET-762 & -0.4020 & $5.08 \times 10^{-21}$ & 587 \\
\hline Pancancer & $K D M 2 B$ & Tubastatin A & -0.4011 & $5.97 \times 10^{-21}$ & 587 \\
\hline BREAST & GADD45A & Refametinib & -0.8026 & 0.0002 & 40 \\
\hline MATBCL & BM/1 & 5-Fluorouracil & -0.7900 & 0.0440 & 27 \\
\hline $\mathrm{SCLC}$ & $A P O B E C 3 A$ & GSK1070916 & 0.7764 & 0.0399 & 29 \\
\hline BREAST & APOBEC $3 C$ & Cetuximab & -0.7278 & 0.0242 & 38 \\
\hline BREAST & APOBEC3G & Cetuximab & -0.7105 & 0.0399 & 38 \\
\hline BREAST & GADD45A & Trametinib & -0.7012 & 0.0399 & 39 \\
\hline NSCLC & $\mathrm{IDH} 1$ & (5Z)-7-Oxozeaenol & 0.5171 & 0.0242 & 91 \\
\hline
\end{tabular}

All agents listed in table were from the GDSC dataset. Abbreviations for cancer categories are provided in Table S2 and in the list of abbreviations. $\rho$, Spearman correlation coefficient; $p_{\mathrm{FDR}}$, FDR adjusted $p$-value; Sample size, the number of available cell lines in each category with available RNA-seq expression data and drug response data 


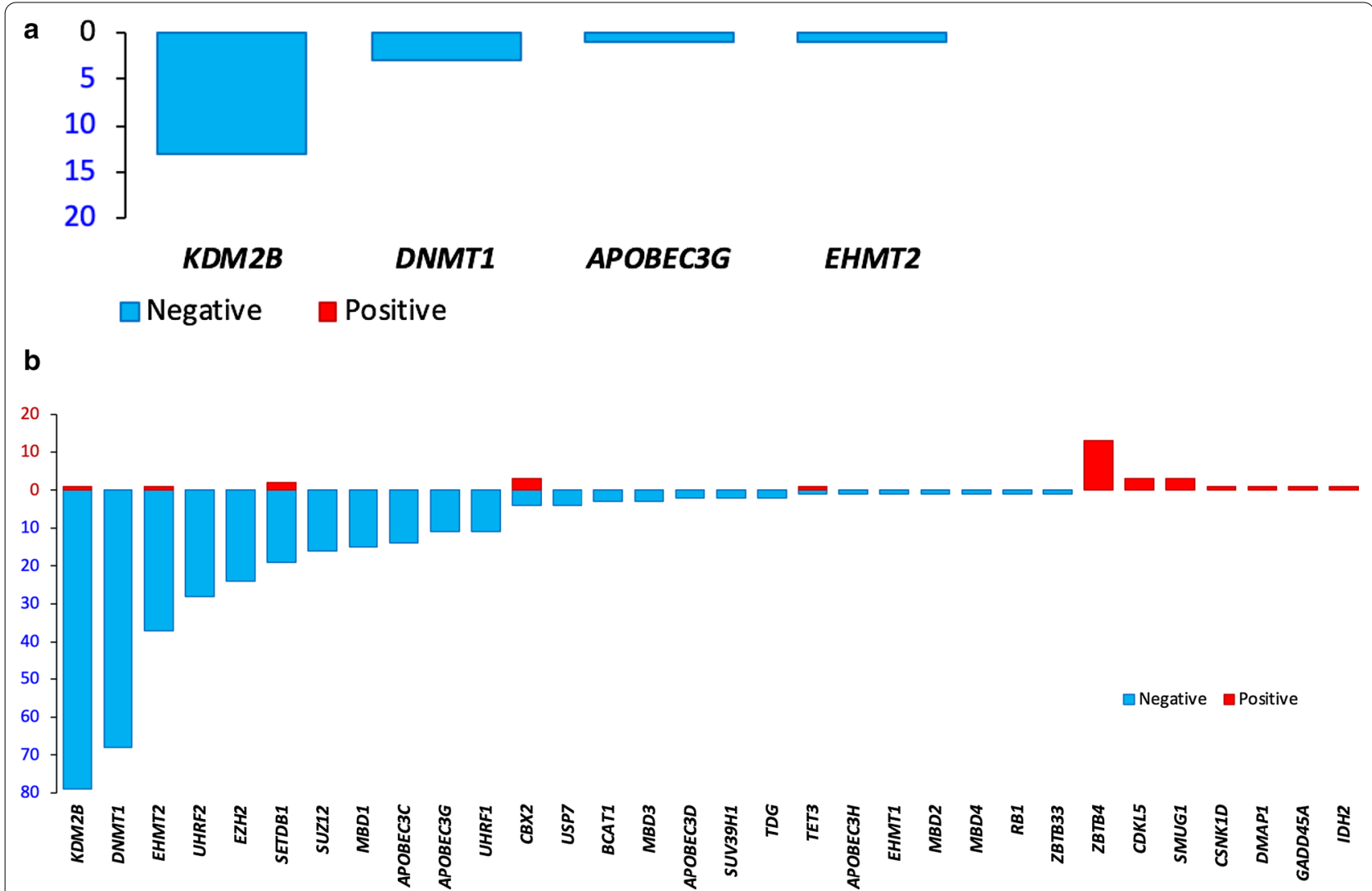

Fig. 3 Numbers of significant correlations of GMD expression with $\log (\mathrm{IC} 50)$ measures of drug response satisfying $p_{\mathrm{FDR}}<0.05$. POsitive correlations are shown as red bars directed upward, whereas negative correlations are shown as blue bars directed downward. A. Correlations satisfying Spearman $|\rho|>0.4$. B. Correlations satisfying Spearman $|\rho|>0.3$

sensitivity. Expression of many GMDs, e.g., $K D M 2 B$, DNMT1, EZH2, SETDB1, SUZ12, SUV39H1, EHMT1, EHMT2, BCAT1, MBD1, MBD2, MBD3, UHRF1, UHRF2, USP7, TDG, APOBEC3C, APOBEC3D, APOBEC3G, and $A P O B E C 3 H$, was predominantly associated with drug sensitivity (Fig. 3; Additional file 3: Table S3). In contrast, ZBTB4, SMUG1, and CDKL5 expression was predominantly associated with drug resistance.

Many epigenetic drugs were associated with GMD expression. Increased expression of the histone demethylase $K D M 2 B$ gene was associated with sensitivity to the HDACi vorinostat, tubastatin A, panobinostat, belinostat, CAY10603, VNLG/124, and AR-42, the bromodomain inhibitor I-BET-762, the SIRT1 inhibitor selisistat, the EHMT1/EHMT2 inhibitor UNC0638, and the DOT1L protein methyltransferase inhibitor SGC0946 (Table 1; Additional file 3: Table S3). Correlations of $K D M 2 B$ expression with agents targeting histone modifications likely involve the epigenetic role of KDM2B and its role in gene regulation [98].

Expression of the maintenance DNA methyltransferase DNMT1 gene was associated with many epigenetic agents, including the HDACi tubastatin A, belinostat, VNLG/124, CAY10603, CUDC-101, and AR-42, the SIRT1 inhibitor selisistat, the EHMT1/EHMT2 inhibitor UNC0638, and the DOT1L inhibitor SGC0946 (Table 1; Additional file 3: Table S3). Their effects on DNMT1 may directly influence DNA methylation. For example, HDACi downregulate gene expression and protein levels of DNMT1, decrease its interaction with PRC2, and induce global DNA hypomethylation [50-52, 54, 55], and DNMT1 is a deacetylation target of SIRT1, which is inhibited by selisistat [29].

Expression of the H3K27 histone methyltransferase $E Z H 2$ gene was modestly $(-0.4 \leq \rho<-0.3$; Additional file 3: Table S3) associated with sensitivity to the HDACi vorinostat, tubastatin $\mathrm{A}$, belinostat, the bromodomain inhibitor I-BET-762, and the DOT1L inhibitor SGC0946. Similar to $K D M 2 B$ and DNMT1, EZH2 associations with epigenetic drugs may involve direct interactions. EZH2, an important regulator of cancer gene expression [99], interacts with class I HDACs [100], and its protein levels and interaction with DNMT1 are downregulated by HDAC inhibitors $[50,55]$. The weak but significant 
$\left(\rho=-0.3028, p_{\mathrm{FDR}}=4.59 \times 10^{-12}\right)$ association of EZH2 expression with sensitivity to I-BET-762 is surprising, as earlier reports found that EZH2 loss increased tumor sensitivity to bromodomain inhibitors [101, 102].

We observed many associations of sensitivity to HDACi and the bromodomain inhibitor I-BET-762 with elevated expression of a number of GMDs, e.g., SETDB1, EHMT2, SUZ12, MBD1, UHRF2, and TDG (Additional file 3: Table S3). $M B D 1$ expression was associated with sensitivity to the EHMT1/EHMT2 inhibitor UNC0638. In contrast, ZBTB4 and GADD45A expression was associated with resistance to HDACi. Associations with pretreatment expression of multiple GMDs are in agreement with the multifaceted actions of epigenetic agents which affect multiple molecular components $[1,100,103]$.

In addition to epigenetic drugs, many GMDs were associated with other categories of antitumor agents (Table 1; Additional file 3: Table S3). Some correlations are directly related to their mechanisms of action. For example, $R B 1$ expression was correlated with sensitivity to palbociclib, a cyclin-dependent kinase (CDK) 4/6 inhibitor $\left(\rho=-0.3060, \quad p_{\mathrm{FDR}}=8.60 \times 10^{-11}\right.$; Additional file 3: Table S3), in agreement with sensitivity of Rb-positive cells to CDK 4/6 inhibitors, which target the cyclin D-CDK 4/6-Rb pathway, and with reduced $R B 1$ expression in cell lines resistant to palbociclib [104-106].

Other associations suggest indirect involvement of the epigenetic pathways in drug response. Elevated expression of KDM2B, DNMT1, EHMT2, and UHRF1 was associated with sensitivity to daporinad, a nicotinamide phosphoribosyltransferase inhibitor (Table 1; Additional file 3: Table S3). KDM2B, DNMT1, EZH2, UHRF1, and $M B D 1$ were associated with sensitivity to the endotelin receptor A inhibitor zibotentan. Expression of $K D M 2 B$, DNMT1, EHMT2, EZH2, SUZ12, MBD1, UHRF2, and $S E T D B 1$ was associated with sensitivity, and that of ZBTB4 with resistance to the RIPK inhibitor XMD13-2 (Table 1; Additional file 3: Table S3). As sample sizes in individual tumor types were modest (Additional file 2: Table S2), many associations were significant in the pancancer analysis only. Their strength could be influenced by the differences in GMD expression and drug response among cancer types. Pancancer associations may indicate the GMD importance in response to the agents with similar activity across different tumor types.

\section{Association of GMD expression with DNA methylation of epigenome targets}

In order to examine indirect modulation of drug response by GMDs via their influence on DNA methylation, we identified their genome methylation targets which were strongly and significantly associated with their expression (Fig. 2b).

\section{Pancancer analysis of individual target probes and gene regions}

Additional files 4, 5: Tables S4 and S5 provide the lists of strong significant pancancer associations of expression of 72 GMDs with DNA methylation. Analysis of 424,840 probes identified 1,905 strong GMD-probe correlations with $p<1.389 \times 10^{-8}$ and $|\rho|>0.5$ (Additional file 4: Table S4). They included 1770 highly significant GMDprobe trans-correlations involving target probes in other genes $\left(0.5<|\rho| \leq 0.7281,1.57 \times 10^{-107} \leq p \leq 3.75 \times 10^{-42}\right)$, which included 19 GMDs and 1,095 probes in 595 target genes. Analysis of gene regions identified 249 strong and significant correlations with GMD expression $\left(p_{\mathrm{FDR}}<0.05,|\rho|>0.5\right)$, including 236 trans-correlations, which involved 17 GMDs and 130 target genes $(0.5<$ $|\rho| \leq 0.6719,1.27 \times 10^{-79} \leq p_{\mathrm{FDR}} \leq 1.08 \times 10^{-37}$; Additional file 5: Table S5).

Among trans-correlations, expression of BCAT1, CBX1, CBX2, DNMT1, DNMT3A, DNMT3B, EHMT1, EHMT2, EZH2, IDH2, KDM2B, MGMT, SETDB1, TDG, TET1, and TET3 was nearly exclusively positively associated with methylation of probes or gene regions (Table 2; Fig. 4; Additional files 6, 7: Tables S6 and S7). Expression of $A P O B E C 3 C, I D H 1$, and $Z B T B 38$ was exclusively, and that of $A P O B E C 3 G$ was predominantly negatively strongly associated with DNA methylation of other genes (Table 2; Fig. 4; Additional files 6, 7: Tables S6 and S7). $M B D 1$ was involved in a small number of both positive and negative correlations (Additional files 6, 7: Tables S6 and S7).

Among GMDs involved in trans-correlations with $\geq 4$ probes and $\geq 5$ gene regions (Table 2; Fig. 4), SETDB1, $C B X 2, K D M 2 B$, and TET3 each had many positive associations with $280-422$ probes and 39-54 gene regions. MGMT, TDG, EHMT2, TET1, EZH2, IDH2, and MBD1 each had positive correlations with $\geq 4$ probes (Table 2; Fig. 4). ZBTB38 and APOBEC3G were involved only in negative trans-correlations with 86 and 63 probes, respectively, and with 4 regions each. $A P O B E C 3 C$ was predominantly involved in negative trans-correlations (Table 2; Fig. 4). All counts of the cis- and trans-correlations of GMD expression with probes and regions are listed in Additional files 6, 7: Tables S6 and S7.

Expression of all DNA methyltransferase genes had positive trans-correlations with DNA methylation. DNMT3A was strongly and significantly associated with 43 probes and 9 regions, DNMT1 with 9 probes and 7 regions, and DNMT3B with 6 probes (Table 2; Fig. 4; Additional files 4, 5, 6, 7: Tables S4-S7), consistent with their functional roles. Interestingly, expression of hydroxymethylating genes was also positively associated with probe methylation, with 280 positive correlations for TET1 and 9 for TET3. While their products 
Table 2 GMDs with the highest numbers of strong transcorrelations between their expression and methylation levels of target individual probes and gene regions in pancancer analysis

\begin{tabular}{|c|c|c|}
\hline GMD & Positive correlations & $\begin{array}{l}\text { Negative } \\
\text { correlations }\end{array}$ \\
\hline \multicolumn{3}{|c|}{ GMDs correlated with $\geq 5$ individual probes } \\
\hline SETDB1 & 422 & 1 \\
\hline$C B \times 2$ & 383 & 0 \\
\hline$K D M 2 B$ & 352 & 1 \\
\hline TET3 & 280 & 0 \\
\hline DNMT3A & 43 & 0 \\
\hline MGMT & 39 & 0 \\
\hline$T D G$ & 20 & 0 \\
\hline DNMT1 & 11 & 0 \\
\hline EHMT2 & 10 & 0 \\
\hline TET1 & 9 & 0 \\
\hline DNMT3B & 6 & 0 \\
\hline$E Z H 2$ & 5 & 0 \\
\hline $\mathrm{IDH} 2$ & 5 & 0 \\
\hline MBD1 & 4 & 1 \\
\hline ZBTB38 & 0 & 86 \\
\hline APOBEC3G & 0 & 63 \\
\hline APOBEC $3 C$ & 2 & 24 \\
\hline \multicolumn{3}{|c|}{ GMDs correlated with $\geq 4$ gene regions } \\
\hline SETDB1 & 54 & 0 \\
\hline$K D M 2 B$ & 53 & 0 \\
\hline TET3 & 41 & 0 \\
\hline$C B \times 2$ & 39 & 0 \\
\hline DNMT3A & 9 & 0 \\
\hline DNMT1 & 7 & 0 \\
\hline APOBEC3G & 0 & 12 \\
\hline ZBTB38 & 0 & 4 \\
\hline APOBEC $3 C$ & 0 & 4 \\
\hline
\end{tabular}

Listed are the counts of correlations satisfying $p<1.389 \times 10^{-8}$ and Spearman $|\rho|>0.5$ for individual probes and $p_{\mathrm{FDR}}<0.05$ and Spearman $|\rho|>0.5$ for gene regions

are involved in oxidation of $5-\mathrm{mC}$ to $5-\mathrm{hmC}$, which subsequently leads to DNA demethylation [13, 107], there have been both reports of the epigenome-wide TET effects on DNA hypomethylation and on increased methylation [108]. The DNA methylation microarray data used in our study did not distinguish between $5-\mathrm{mC}$ and $5-\mathrm{hmC}[88,109]$, and positive associations of TET3 and TET1 (Table 2; Fig. 4; Additional files 4, 5, 6, 7: Tables S4-S7) likely involve measures of a mixture of 5-mC and 5-hmC.

GMD expression was associated with methylation of probes and regions in many important cancer genes (Additional files 4, 5: Tables S4 and S5). Selected examples of such associations are discussed in detail in
Additional file 19: Data S1. For example, we observed epigenetic regulation of methylation of probes and/or gene regions of $A B L 1, A B L 2, M E T, X R C C 5$, KIFC3, and TIMP. Similarly, we observed associations of expression of multiple GMDs with a probe in TGFBI, whose product has been associated with poor prognosis in colorectal cancer and is a predictive biomarker for dasatinib sensitivity [110, 111]. Among the $A B C$ family transporter genes, methylation of $A B C C 1$ and $A B C C 3$ was associated with GMD expression, suggesting multiple epigenetic pathways of their regulation.

Expression of multiple GMDs was associated with methylation of many probes and regions in genes involved in inflammation, e.g., IRAK2 which encodes an activator of the NF- $\mathrm{KB}$ pathway, and tumor necrosis factor receptor genes TNFRSF10B and TNFRSF1A. Among the Hippo pathway components, methylation of WWTR1 $(T A Z)$ and TEAD1 was significantly correlated with GMD expression (Additional files 4, 5: Tables S4 and S5; Additional file 19: Data S1).

GMD expression was correlated with methylation of other genes involved in epigenetic processes or global transcriptional regulation (Additional files 4, 5: Tables S4 and S5; Additional file 19: Data S1). Expression of the histone methyltransferase SETDB1 and histone lysine demethylase $K D M 2 B$ genes was positively correlated with methylation of the histone deacetylase $H D A C 9$, suggesting $H D A C 9$ regulation by SETB1 and KDM2B or coregulation among different histone modifiers. $K D M 2 B$ and TET3 expression was positively associated with methylation of $F T O$, which participates in RNA methylation [13]. TET3 and SETDB1 expression was positively correlated with methylation of NNMT, whose product promotes tumorigenesis and regulates the availability of methyl groups for cellular methylation reactions [112, 113]. MED1 and MED20 methylation was correlated with multiple GMDs, suggesting a potential influence of GMD expression on global transcriptional regulation. MED1 and MED20 are subunits of the mediator of RNA polymerase transcription. They participate in the Mediator complex, which is involved in transcriptional regulation of RNA polymerase II-dependent genes [114].

We also observed strong and highly significant ciscorrelations of expression of 11 GMDs with their own probes and regions (Additional files 4, 5: Tables S4 and S5). Expression of $A P O B E C 3 C, A P O B E C 3 D, B C A T 1$, CBX2, DNMT3A, MGMT, IDH2, PHC2, TET1, and $Z B T B 38$ had 106 positive and 29 negative cis-correlations with methylation of their own 135 probes (Additional file 4: Table S4). Expression of $A P O B E C 3 B, A P O B E C 3 C$, $A P O B E C 3 D, B C A T 1, M G M T$, and PHC2 was associated with methylation of their gene regions (Additional file 5: Table S5). The majority of cis-associations of the probe 


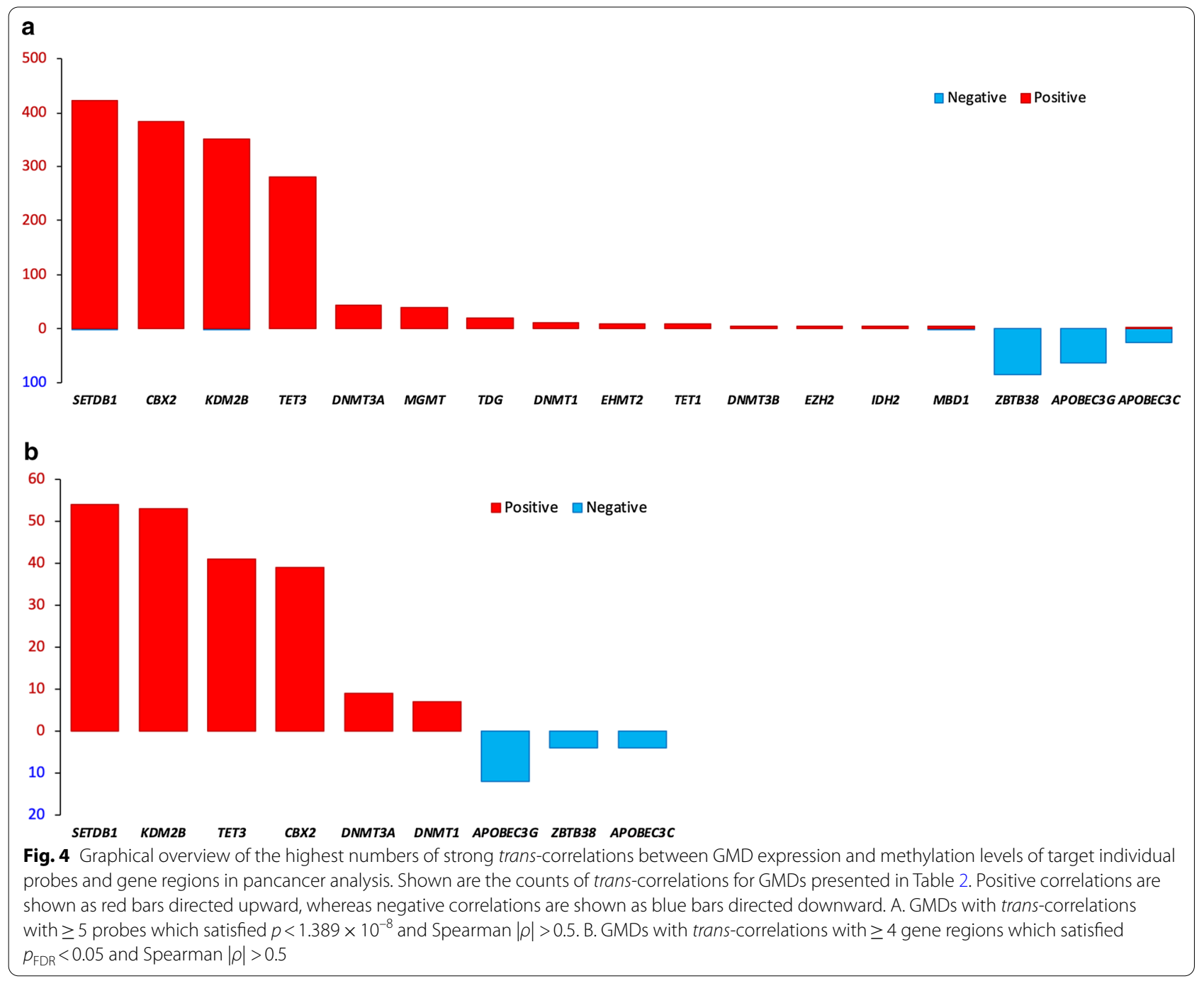

and region methylation of GMDs with their expression was strongly negative (Additional files 4, 5, 6, 7: Tables S4-S7), suggesting regulation of expression of these GMDs by their promoter methylation. Consistent with the well-documented repressive effect of the MGMT promoter methylation on its expression [37], methylation of the $5^{\prime}$ UTR, the 1st exon, and several individual probes of $M G M T$ was negatively correlated with its expression.

\section{Analysis of individual cancer categories}

We observed strong and significant correlations of GMD expression with methylation of probes and gene regions in the stratified analysis among cancer categories. The use of the threshold of $p<6.039 \times 10^{-10}$ for the probes identified 372 very strong correlations with $0.5801 \leq|\rho| \leq 1$ (Additional file 8: Table S8), including 259 trans-correlations between 44 GMDs and probe methylation in 166 target genes. They represent the strongest and highly significant associations of GMD expression with individual probes. Methylation of many other probes was also correlated with GMD expression but did not satisfy the stringent $p$-value threshold (data not shown). Correlation analysis of GMD expression with methylation of gene regions identified 14,609 associations with $0.5<$ $|\rho| \leq 1$ and $p_{\text {FDR }}<0.05$, including 14,558 trans-correlations between expression of all 72 GMDs and the gene regions in 8,336 target genes (Additional file 9: Table S9).

Expression of many GMDs was correlated with methylation of multiple probes and gene regions (Table 3). A large number of associations was observed in chronic leukocytic leukemia (CLLE; Additional file 21: Fig. S2; Additional file 11: Table S11). Among the GMDs associated with $\geq 10$ gene regions in CLLE, multiple positive correlations were observed for UHRF1 (1320 positive associations), CBX2 (687), PHC2 (651), CSNK1E (536), EHMT2 (392), SUV39H2 (293), IDH2 (165), DNMT1 
Table 3 GMDs with the highest numbers of strong correlations between their expression and methylation levels of target probes and gene regions within individual cancer categories

\begin{tabular}{|c|c|c|c|}
\hline Cancer type & GMD & $\begin{array}{l}\text { Positive } \\
\text { correlations }\end{array}$ & $\begin{array}{l}\text { Negative } \\
\text { correlations }\end{array}$ \\
\hline \multicolumn{4}{|c|}{ GMD correlations with $\geq 5$ individual probes } \\
\hline NSCLC & MGMT & 25 & 0 \\
\hline CLLE & UHRF1 & 15 & 0 \\
\hline NSCLC & DNMT3A & 12 & 0 \\
\hline NSCLC & PHC2 & 2 & 9 \\
\hline NSCLC & $C B \times 2$ & 8 & 2 \\
\hline BREAST & EHMT1 & 6 & 0 \\
\hline THCA & $C B \times 2$ & 6 & 0 \\
\hline CLLE & SUV39H2 & 5 & 0 \\
\hline COAD/READ & $A P O B E C 1$ & 0 & 34 \\
\hline CLLE & ZBTB38 & 0 & 25 \\
\hline BREAST & SMUG1 & 2 & 5 \\
\hline NSCLC & SETDB1 & 0 & 7 \\
\hline \multicolumn{4}{|c|}{ GMD correlations with $>100$ gene regions } \\
\hline CLLE & UHRF1 & 1320 & 2 \\
\hline CLLE & $C B \times 2$ & 687 & 0 \\
\hline CLLE & PHC2 & 651 & 0 \\
\hline CLLE & CSNKIE & 536 & 0 \\
\hline CLLE & EHMT2 & 392 & 0 \\
\hline COAD/READ & SIRT1 & 359 & 0 \\
\hline BREAST & EHMT1 & 268 & 39 \\
\hline CLLE & SUV39H2 & 293 & 1 \\
\hline SCLC & DNMT1 & 261 & 1 \\
\hline STAD & TET1 & 239 & 0 \\
\hline CLLE & $\mathrm{IDH} 2$ & 165 & 0 \\
\hline CLLE & DNMT1 & 154 & 0 \\
\hline SARCOMA & IDH2 & 139 & 5 \\
\hline STAD & $C B \times 1$ & 135 & 1 \\
\hline COAD/READ & SUZ12 & 126 & 1 \\
\hline CLLE & DNMT3B & 120 & 0 \\
\hline GLIOMA & TET3 & 113 & 0 \\
\hline COAD/READ & UHRF1 & 111 & 0 \\
\hline CLLE & $E Z H 2$ & 106 & 0 \\
\hline BREAST & BCAT1 & 89 & 63 \\
\hline CLLE & ZBTB38 & 0 & 869 \\
\hline COAD/READ & $A P O B E C 1$ & 5 & 766 \\
\hline BREAST & SMUG1 & 34 & 273 \\
\hline BREAST & $T D G$ & 50 & 195 \\
\hline BREAST & APOBEC $3 C$ & 22 & 97 \\
\hline
\end{tabular}

Listed are the counts of correlations satisfying $p<6.039 \times 10^{-10}$ and Spearman $|\rho|>0.5$ for individual probes and $p_{\mathrm{FDR}}<0.05$ and Spearman $|\rho|>0.5$ for gene regions

(154), DNMT3B (120), EZH2 (106), TRIM28 (56), PCNA (48), SETDB1 (26), UHRF2 (21), DNMT3A (19), BMI1 (19), MBD3 (16), and SUV39H1 (14). Negative associations with $\geq 10$ gene regions in CCLE cell lines were observed for ZBTB38 (869), EED (47), CDKL5 (33), $M B D 2$ (32), APOBEC3A (17), AIDCDA (15), and $A P O B E C 3 B$ (11). Consistent with their roles in DNA methylation and with the direction of associations in the pancancer dataset, DNTM1, DNMT3A, and DNTM3B expression was associated with increased methylation of many individual probes and gene regions in multiple tumor types (Table 3; Additional file 21: Fig. S2; Additional files 10, 11: Tables S10 and S11). Expression of UHRF1, whose product has multiple roles in DNA methylation including interactions with DNMT1, DNMT3a, DNMT3b and G9a, control of DNMT1 abundance, and targeting DNMT1 to hemimethylated DNA during replication (Additional file 1: Table S1) [24, 25, 32, 40, 115], was strongly associated with methylation of multiple probes and regions in many tumor types (Table 3; Additional file 21: Fig. S1; Additional files 10, 11: Tables S10 and S11). While UHRF1 associations did not reach $|\rho|>0.4$ in the pancancer data (Fig. 4; Additional files 6, 7: Tables S6 and S7), its expression had weaker positive significant associations with 12,611 probes with $p<1.389 \times 10^{-8}$ and $0.3<\rho<0.4$ and only 10 negative correlations with $p<1.389 \times 10^{-8}$ and $-0.4<\rho<-0.3$ (data not shown). This highlights the importance of URHF1 in DNA methylation in tumors.

Associations of some GMDs were specific to individual cancer categories, suggesting heterogeneity of the mechanisms and of the strength of epigenetic interactions among cancer histologies. For example, in CLLE, ZBTB38 expression was significantly $\left(p_{\mathrm{FDR}}<0.05\right)$ negatively correlated with $\rho<-0.5$ with 869 regions of other genes and had no positive trans-associations. By contrast, when using this threshold, ZBTB38 had only 3 negative and 1 positive correlations with gene regions in NSCLC and only 1 negative correlation in breast cancer cell lines (Additional file 21: Fig. S2; Additional file 11: Table S11). It had no negative trans-correlations and 1 and 11 positive trans-correlations with gene regions in the COAD/ READ and PAAD categories, respectively. Many other GMDs also had variable numbers of positive and negative strong associations in different tumors (Table 3; Additional file 21: Fig. S2; Additional files 8, 9, 10, 11: Tables S8-S11).

Similar to the pancancer analysis, we observed multiple strong significant associations of GMD expression with methylation of other GMDs and other genes involved in epigenetic processes and chromatin structure, maintenance, and regulation. For example, $A P O B E C 2$ expression in bladder cancer was associated with methylation of the $5^{\prime}$ UTR and the 1st exon of the DNA demethylase $A L K B H 2$, whose product removes $\mathrm{N}^{1}$-meA and $\mathrm{N}^{3}$-meC ( $\rho=-0.8687$; Additional files 1, 9: Tables S1 and S9) [38, 39]. 
We observed many strong tumor type-specific significant correlations of GMD expression with methylation of genes important in cancer. The detailed results are presented in Additional files 8, 9: Tables S8 and S9, and selected examples are discussed in Additional file 19: Data S1. They include associations of methylation of regions of the $A B L 2$ oncogene in breast cancer and in $\mathrm{COAD} / \mathrm{READ}$, and of the epidermal growth factor receptor EGFR gene in CLLE. Upstream gene regions of the tumor suppressor $R U N X 1$ were positively correlated with DNMT3A expression in liver hepatocellular carcinoma. RUNX1 is downregulated in the early stages in hepatocellular carcinoma [116], and our findings suggest a potential role of DNA methylation in its regulation. The upstream region of $M Y C N$, which may play a regulatory role in $M Y C N$ expression $[86,117]$, was associated with GMD expression in breast cancer and in COAD/READ. In CLLE, the body of EGFR was strongly positively correlated with $E H M T 2$ and $P H C 2$.

In several tumor types, GMD expression was associated with methylation of the regions of $M L K L$ and RIPK3 encoding key players in necroptosis [118], RIPK2 and RIPK4, which are involved in inflammatory signaling and NF- $\mathrm{kB}$ activation $[119,120]$, and IRAK2, IRAK3, and IRAK4, which mediate the toll-like receptor and interleukin-1 receptor signaling pathways and are involved in the NF- $\kappa B$ activation [121] (Additional file 9: Table S9; Additional file 19: Data S1). Numerous GMDs were strongly associated with methylation of components of the TNF- $\alpha$ signaling pathway [122] including TNF and other TNF family members, e.g., TNSF11 (RANKL) and TNSF13B $(B A F F)$ involved in activation of NF-kB signaling [123], TNFAIP 3 and TNFAIP8L 2 encoding TNF- $\alpha$ induced proteins, and the TNF receptor superfamily members.

GMD expression was strongly correlated with methylation of multiple components of the Hippo signaling pathway [122] (Additional files 8, 9: Tables 8 and S9; Additional file 19: Data S1), including YAP1 in stomach adenocarcinoma (STAD), WWTR1 (TAZ) in STAD, NSCLC, breast cancer, sarcoma, CLLE, and COAD/ READ, TEAD1 in CLLE, TEAD2 in glioma and SCLC, LATS1 in CLLE, LATS2 in mature B-cell lymphoma, and MST1 in CLLE. Methylation of RASSF1, RASSF2, RASSF3, RASSF6, RASSF7, and RASSF9, from the RASSF regulator family [122] was strongly associated with expression of several GMDs in a variety of cancer categories. As discussed above, WWTR1 and TEAD1 methylation was also associated with GMD expression in the pancancer data. These findings indicate a strong epigenetic regulation of the Hippo pathway.

We also found extensive epigenetic regulation of genome integrity. GMD expression was associated with methylation of RAD51, RAD51C, RAD50, RAD1,
RAD9A, RAD9B, RAD18, RAD21L1, and RAD23A (Additional file 9: Table S9; Additional file 19: Data S1). RAD proteins are involved in DNA repair, chromosomal segregation, and checkpoint control [124-127]. We observed associations of methylation of RAD51 in glioma, and of $R A D 51 C$ and $R A D 50$ in CLLE. Upstream regions of $X R C C 2, X R C C 5$, and $X R C C 6$ were also positively associated with multiple GMDs. Methylation of the tumor suppressor gene TP53BP1, whose product mediates DNA damage response, was associated with GMD expression in COAD/READ, bladder cancer, and SCLC.

In several tumor categories, methylation of TMEM173 (STING), TREX1, and C6orf150 (cGAS) was correlated with GMD expression (Additional file 9: Table S9; Additional file 19: Data S1). Their products regulate the cytosolic DNA-sensing cGAS-STING innate immune pathway, activation of which is associated with improved tumor response to drug treatment and immunotherapy [128-134]. Our results suggest epigenetic influences on its regulation. Similarly to individual cancer types, we observed weaker significant $(0.3001<|\rho| \leq 0.4124$, $7.00 \times 10^{-28} \leq p \leq 6.90 \times 10^{-15}$ ) pancancer associations of upstream regions of these genes with multiple GMDs (data not shown).

\section{Association between methylation of target probes and gene regions and drug response}

After identifying 1,306 target probes in 45 genes and 11,754 gene regions in 8,374 genes, which were strongly and significantly associated with GMD expression in pancancer analysis or in individual cancer types (Additional files 4, 5, 8, 9: Tables S4, S5, S8, and S9), we examined the association of their methylation with $\log$ (IC50) (Fig. 2). Only 4 probes and 3 regions had significant correlations with $|\rho|>0.5$ in the pancancer data $\left(p_{\mathrm{FDR}} \leq 2.94 \times 10^{-14}\right.$ for the probes and $p_{\mathrm{FDR}} \leq 9.26 \times 10^{-}$ 17 for the gene regions; Additional files 12, 13: Tables S12 and S13). The probe cg16411668 in a non-coding region was associated with $K D M 2 B, D N M T 3 A$, and SETDB1 expression and with panobinostat sensitivity $(\rho=-0.5245)$. The probes cg08422793 and cg20824939 in intergentic regions and cg20092122 in BST2, the bone marrow stromal antigen 2 , were associated with sunitinib resistance $(0.5027 \leq \rho \leq 0.5162)$ and with $A P O B E C 3 G$ and/or $A P O B E C 3 C$ expression. Consistent with the cg20092122 association, the TSS1500, TSS200, and the 1st exon of BST2 were also associated with sunitinib resistance $(\rho=0.5167, \rho=0.4622$ and 0.4507 ; Additional file 13: Table S13). All these upstream regions were associated with $C B X 2$ expression. The $5^{\prime} \mathrm{UTR}$ of SELPLG was also associated with sunitinib resistance $(\rho=0.5305)$, and with $H D A C 1$ expression. The CYR61 body was associated 
with panobinostat sensitivity $(\rho=-0.5021)$ and with $K D M 2 B$ and $S E T D B$ expression.

Using a less stringent cutoff of $|\rho|>0.4$ for significant pancancer associations with $\log (\mathrm{IC} 50)$, we found 1,213 probe correlations and 714 gene region correlations (Additional files 12, 13: Tables S12 and S13) including many genes involved in cancer progression or drug transport. Examples of probe associations included $A B C$ family transporters $A B C C 1$ and $A B C C 3, S L C$ transporters SLC2A1, SLC4A7, SLC22A5, SLC25A22, SLC26A1, SLC39A11, SLC39A13, and SLC45A1, the oncogenes $A B L 1, A B L 2$, NF1, and RPTOR, the DUSP5 and DUSP14 kinase genes, RAD51L1 involved in homologous recombination repair [135], FTO and HDAC9 encoding epigenetic factors, IRAK2, MAP3K14, KIF3, ubiquitin related genes NEURL3 and UBE2O, and NFIA encoding the tumor-promoting transcription factor nuclear factor IA [136] (Additional file 12: Table S12). Associations of gene regions included the ERBB2 (HER2) and NOTCH3 oncogenes, the tumor suppressor PHLDA1, CASP8 which plays a central role in apoptosis $[118,122]$, the N-myc interactor NMI, PON2, CAMKK2, LIPG, the DUSP6 kinase gene, KIF12, the E-cadherin gene $C D H 1$, the histone acetyltransferase MYST1 (KAT8, or MOF), ubiquitin related NDFIP2 and $U B A 7$ [137, 138], NR1D2 (Rev-erb $\beta$ ) encoding a transcriptional repressor, $R A P$ $1 G A P 2, R A S E F$, the glucocorticoid receptor gene NR3C1, $P P A P 2 C$, and the $S L C$ transporter genes SLC44A2 and PQLC3 (SCL66A3) (Additional file 13: Table S13).

We observed modest $(|\rho|>0.4)$ significant correlations involving both probes and the entire regions of many important genes (Additional files 12, 13: Tables S12 and S13). The $5^{\prime}$ UTR, 1st exon, and multiple probes in the tumor suppressor gene DAPK3 were associated with the HDACi vorinostat and panobinostat. Panobinostat sensitivity was also correlated with methylation of a probe and the entire TSS1500 in NNMT, which controls the methylation potential of tumor cells [112], consistent with NNMT upregulation in a panobinostat resistant glioma cell line [139] and with the correlation of NNMT expression with vorinostat resistance [140]. Individual probes and the body of the oncogene $D D A 1$ were associated with sensitivity to the HDACi vorinostat and panobinostat, the bromodomain inhibitor I-BET-762, the PDK1 inhibitor BX-912, the LXR agonist T0901317, and the HER2 inhibitor TL-2-105 (Additional files 12, 13: Tables $\mathrm{S} 12$ and S13). The 5'UTR and its probes in RUNX1 were associated with resistance to sunitinib, cyclopamine, and Z-LLNle-CHO. The $5^{\prime} \mathrm{UTR}$, the body, and their probes in the transcriptional regulator SP1 gene were associated with resistance to refametinib and tanespimycin. The $5^{\prime}$ UTR and its probes in the transcriptional regulator $M A F K$ gene were associated with sensitivity to the
IKK inhibitor BMS-345541, the CRAF inhibitor TL-2105 , and the HDACi vorinostat. Many MAFK probes were also associated with other agents. The TSS200 of TREX1 was associated with vorinostat sensitivity $\left(\rho=-0.4101, \quad p_{\mathrm{FDR}}=5.28 \times 10^{-19}\right)$, and the TSS200 of TMEM173 (STING) was associated with sunitinib resistance $\left(\rho=0.4202, p_{\mathrm{FDR}}=5.09 \times 10^{-9}\right)$.

Methylation of many probes and regions was significantly associated with expression of $K D M 2 B, S E T D B 1$, CBX2, EHMT1, DNTM1, DNMT3A, DNMT3B, TET1, TET2, TET3, MBD1, SMUG1, ZBTB38, APOBEC1, $A P O B E C 3 C, A P O B E C 3 G$, and other GMDs, suggesting that GMDs may influence drug response via methylation of epigenome targets. Many probes correlating with drug response were associated with expression of multiple GMDs, suggesting intertwined pathways of epigenetic regulation.

Within tumor types, 904 probe-drug and 630 gene region-drug associations were strong and significant $\left(|\rho|>0.5, p_{\mathrm{FDR}}<0.05\right.$; Additional files 14, 15: Tables S14 and S15). Many of the same probes and regions also had weaker correlations with similar agents in the pancancer data (Additional files 12, 13: Tables S12 and S13). For example, cg25928474 in the $A B C C 3$ transporter gene had strong correlations with sensitivity to the HDACi AR-42 in ALL ( $\rho=-0.9636$; Additional file 14: Table S14) and panobinostat in pancancer data $(\rho=-0.4189$; Additional file 12: Table S12). It had weaker $(0.4<|\rho|<0.3)$ significant pancancer correlations with sensitivity to the HDACi vorinostat and the bromodomain inhibitor I-BET-762, and with other agents (data not shown). Its methylation was associated with expression of multiple GMDs including TET3, TDG, SETDB1, ZBTB38, $K D M 2 B$, and $C B X 2$. Multiple probes and regions in other target genes, e.g., $A B L 2, S P 1, D A P K 3, N F 1$, IRAK2, $U B E 2 O$, and FTO, which were associated with drug response in the pancancer data, also had strong associations in individual tumor types $(|\rho|>0.5$; Additional files 14, 15: Tables S14 and S15). Other examples of significant associations with $\log (\mathrm{IC} 50)$ in specific tumors included WNT3A, WNT7A, FOXO3, FOX3P, WWTR1 (TAZ), TEAD2, TNFRSF1OB, and RASSF7.

\section{Regression analysis of response to trametinib}

After identifying significant methylation probes and gene regions associated with trametinib (Additional files 4, 5: Tables S4 and S5) and of the GMDs whose expression was associated with response to that agent with $|\rho|>0.5$ (Table 1), we included them individually as predictor variables in multivariate regression analysis of trametinib response. We also used the mutation status of $B R A F$, $K R A S$, and $N R A S$ as additional predictor variables. When BRAF V600E and non-synonymous changes in KRAS 
or NRAS were considered, methylation of the $5^{\prime} \mathrm{UTR}$ of C7orf49 and of the probe cg00172872 in the intergenic region on 12q21.33 remained significantly associated with trametinib in pancancer and breast cancer $\left(5.24 \times 10^{-}\right.$

$\left.{ }^{13} \leq p_{\mathrm{FDR}} \leq 0.0023\right)$. BRAF V600E was also highly significant in these models both in breast cell lines and in the pancancer data $\left(p \leq 2.52 \times 10^{-5}\right)$, while the variants in KRAS or NRAS were significant in the pancancer data $\left(p \leq 2.23 \times 10^{-17}\right.$; data not shown). cg00172872 was associated with $C B X 2$, SETDB1, and TET3 expression (Additional files 4, 14: Tables S4 and S14), while the $5^{\prime}$ UTR of C7orf 49 was associated with GADD45A (Additional files 9, 15: Tables S9 and S15). GADD45A expression was also strongly correlated with trametinib response in breast cancer $\left(\rho=-0.7012 ; p_{\mathrm{FDR}}=0.0399\right.$; Table 1$)$. When adding the BRAF V600E, KRAS, and NRAS mutation status to the model, association of GADD45A expression with trametinib in breast cell lines had $p=0.0003$ prior to FDR adjustment, and $p_{\mathrm{FDR}}=0.1566$ after the adjustment (data not shown). These results suggest the importance of the GADD45A expression and C7orf49 methylation in trametinib response. C7orf49 (CYREN) is a cell-cyclespecific inhibitor of classical non-homologous end joining of DNA double-strand break repair, regulating the selection of DNA double-strand repair pathway [141].

\section{Correlations of average epigenome methylation levels with GMD expression and drug response}

Pancancer analysis showed very weak $\left(|\rho|<0.35, p_{\mathrm{FDR}}<0.05\right)$ significant correlations of expression of 43 GMDs with average epigenome methylation (data not shown). The strongest correlations were for HELLS $\left(\rho=0.3356, p_{\mathrm{FDR}}=1.41 \times 10^{-}\right.$ $\left.{ }^{16}\right)$, UHRF1, ZBTB38, and TET3 $(\rho=0.2423,-0.2951$, and 0.2496 , respectively; $\left.4.82 \times 10^{-13} \leq p_{\mathrm{FDR}} \leq 2.37 \times 10^{-9}\right)$. $D N M T 1, D N M T 3 A$, and $D N M T 3 B$ had very weak positive correlations $(\rho=0.1902,0.1886$, and 0.2970 , respectively; $\left.6.63 \times 10^{-9} \leq p_{\mathrm{FDR}} \leq 7.29 \times 10^{-6}\right)$, consistent with their roles in promoting epigenome methylation (Fig. 4; Additional files 6, 7: Tables S6 and S7). Weak pancancer correlations are likely due to the differences in expression and methylation of individual genes among cancer categories.

Associations of epigenome methylation with GMD expression in individual tumor types satisfying $p_{\mathrm{FDR}} \leq 0.15$ are listed in Table 4. Many of them were very strong. Positive associations of CSNK1E in CLLE and CBX2 in LAML reached significance $\left(\rho=0.8750\right.$ and $0.8018, p_{\mathrm{FDR}}=0.0306$; Table 4), consistent with their positive associations with many gene targets in these leukemia types (Additional file 21: Fig. S2; Additional files 10, 11: Tables S10 and S11). CSNK1E, casein kinase $1 \varepsilon$, binds to DNMT1 and phosphorylates it, reducing its DNA-binding activity (Additional file 1: Table S1) [115, 142]. CBX2, a PRC1 member, is found in complexes with DNMT3A and DNMT3B [26,
Table 4 Correlations of average epigenome methylation with GMD expression in individual cancer categories satisfying $p_{\mathrm{FDR}}<0.15$

\begin{tabular}{llcll}
\hline Cancer category & GMD & Spearman $\boldsymbol{\rho}$ & $\boldsymbol{p}_{\text {FDR }}$ & Sample size \\
\hline CLLE & CSNK1E & 0.8750 & $0.0306^{*}$ & 15 \\
LAML & CBX2 & 0.8018 & $0.0306^{*}$ & 19 \\
PAAD & CBX1 & 0.6606 & 0.0538 & 27 \\
COAD/READ & SIRT1 & 0.5506 & 0.0538 & 43 \\
COAD/READ & SUZ12 & 0.5471 & 0.0538 & 43 \\
COAD/READ & HELLS & 0.5388 & 0.0538 & 43 \\
CLLE & CBX2 & 0.8036 & 0.0733 & 15 \\
NSCLC & UHRF1 & 0.3563 & 0.0766 & 96 \\
LAML & MECP2 & 0.7158 & 0.0845 & 19 \\
PAAD & EZH2 & 0.6172 & 0.0845 & 27 \\
GLIOMA & TET3 & 0.5439 & 0.0845 & 36 \\
COAD/READ & SUV39H2 & 0.5115 & 0.0845 & 43 \\
CLLE & DNMT3B & 0.7750 & 0.0886 & 15 \\
CLLE & DNMT1 & 0.7643 & 0.1029 & 15 \\
LIHC & CBX1 & 0.7279 & 0.1029 & 17 \\
GLIOMA & PHC2 & -0.5228 & 0.1119 & 36 \\
CLLE & UHRF1 & 0.7393 & 0.1455 & 15 \\
MATBCL & UHRF2 & 0.5291 & 0.1455 & 33 \\
NSCLC & MBD1 & -0.3169 & 0.1455 & 96 \\
\hline An & &
\end{tabular}

An asterisk (*) indicates statistically significant associations with $p_{\mathrm{FDR}}<0.05$

143]. Positive correlations of $C B X 1$ in PAAD and of SIRT1, SUZ12, and HELLS in COAD/READ were nearly significant $\left(0.5388 \leq \rho \leq 0.5506,0.1886\right.$, and $0.2970 ; p_{\mathrm{FDR}}=0.0538$; Table 4). DNMT1, DNMT3A, and DNMT3B associations were also positive but did not reach statistical significance, with the strongest correlations in CLLE $(\rho=0.7643,0.5286$, and 0.7750).

Pancancer correlations of the average genome methylation with drug response were very weak, even though 14 agents reached significance ( $p_{\mathrm{FDR}}<0.05$; data not shown). The majority of the correlations were weakly negative, suggesting a weak trend for higher sensitivity of more methylated cell lines. Only lapatinib, ZG-10, and WZ-1-84, had $|\rho|>0.2\left(-0.2106 \leq \rho \leq-0.2064 ; p_{\mathrm{FDR}} \leq 0.0447\right.$; data not shown).

Stratified analysis within tumor types identified a strong and significant correlation between epigenome methylation in bladder cancer and sensitivity to the CDK inhibitor THZ-2-49 ( $\rho=-0.8596, p_{\mathrm{FDR}}=0.0243$; data not shown). Two correlations in COAD/READ were strong with $p_{\mathrm{FDR}}<0.15$, including sensitivity to the PDK-1 inhibitor BX795 and the proteasome inhibitor MG-132 $(\rho=-0.6376$ and $-0.8791, p_{\mathrm{FDR}}=0.126$ for both; data not shown). The biological mechanisms of these associations require further investigation. 


\section{Validation of the findings and their clinical significance using independent datasets}

Among pancancer correlations of GMD expression with drug response presented in Table 1, which satisfied Spearman $|\rho|>0.4$ and $p_{\mathrm{FDR}}<0.05$, seven associations had both GMD expression data and $\log (\mathrm{GI} 50)$ drug response data for the same agents available in the NCI-60 dataset in CellminerCDB (Additional file 16: Table S16). Among them, $K D M 2 B$ expression was strongly and highly significantly correlated with sensitivity to the HDAC inhibitor vorinostat in the NCI-60 cancer cell lines (Pearson $r=-0.51, p=4.3 \times 10^{-5}$ ), providing a strong support for our initial finding of this association in the CCLEGDSC dataset. Four additional associations for $K D M 2 B$, $D N M T 1$, and $A P O B E C 3 G$ had the same direction of association between GMD expression and drug sensitivity both in the CCLE-GDSC and NCI-60 datasets, but they did not reach statistical significance in the NCI-60 data (Additional file 16: Table S16).

Additional file 17: Table S17 shows the strength of Pearson correlation in the NCI-60 data from CellminerCDB, used for validation of significant correlations between GMD expression and DNA methylation of upstream gene regions (TSS1500, TSS200, 5'UTR, and the 1st exon) in the pancancer CCLE-GDSC data from Additional file 5: Table S5. Among the 116 significant correlations from CCLE-GDSC data listed in Additional file 17: Table S17 which also had comparable NCI-60 data (GMD expression and gene-averaged methylation derived from the upstream probes) in CellminerCDB, 63 (54\% of the total) had both the Pearson correlation $p<0.05$ in the NCI-60 data and the same direction of association in both datasets, confirming our initial findings. Many additional GMD-target gene associations in Additional file 17: Table S17 had the same direction of correlation both in the CCLE-GDSC and NCI-60 datasets but did not reach statistical significance.

We also observed a strong and consistent confirmation of our findings in an independent NCI SCLC dataset consisting of 66 small cell lung cancer cell lines, which we had generated previously [86]. Additional file 18: Table S18 provides Spearman correlation results between GMD expression and DNA methylation of gene regions in 66 SCLC cell lines. They validate the significant findings in the SCLC category of the CCLE-GDSC data from Additional file 9: Table S9 that had $p_{\mathrm{FDR}}<0.05$ and $|\rho|>0.5$. Among 734 significant GMD-gene region correlations with available data in both datasets, 521 (71\%) had both associations in the same direction and $p<0.05$ in the independent NCI SCLC dataset. Among validated results for multiple associated GMDs in Additional file 18: Table S18, we note multiple correlations involving the KMT2A $(M L L)$ gene which is frequently mutated in SCLC, and $E Z H 2$, an important epigenetic drug target in SCLC, pharmacologic inhibition of which suppresses SCLC growth and chemoresistance [144-146].

We were also able to validate several significant correlations of individual target epigenome probes and gene regions with drug response in the SCLC category in the CCLE-GDSC dataset (Additional files 14, 15: Tables S14 and S15) using the associations in the NCI SCLC dataset, even though these two datasets contained many different agents and used two different Illumina methylation arrays. We used the NCI SCLC dataset to confirm the associations of the TSS1500 of CXCL17 and TSS1500 of PPR18 with response to docetaxel and of the probes cg0260189 in the body of $B I K$ with docetaxel, cg04619882 in the body of KIAAA1324 with dactolisib, and cg04619885 in the body of UBE2O with PD0325901 $(0.2503 \leq|\rho| \leq 0.3623,0.0029 \leq p \leq 0.0427$, and the direction of associations was also identical in both datasets; data not shown). For some agents which were unique to the CCLE-GDSC screen, confirmation of clinically important associations with epigenomic targets may be suggested based on indirect evidence. For example, methylation of the TSS1500 of TEAD2 in the SCLC category of the CCLE-GDSC dataset was associated with resistance to the MTOR inhibitor temsirolimus (Additional file 15: Table S15). It is consistent with an earlier report by an independent group using SCLC CCLE cell lines and with our previous findings in the NCI SCLC dataset, which showed that increased methylation and low expression of the genes encoding TEAD co-activators YAP1 and TAZ in the Hippo pathway in SCLC were associated with resistance to multiple mTOR inhibitors $[86,147,148]$.

We further evaluated the available indirect support for the potential clinical significance of our findings in pancreatic adenocarcinoma, by examining published reports based on patient data. Among the five genes whose probes and/or region methylation was associated with in vitro drug response in our analysis of the PAAD category in the CCLE-GDSC dataset (Additional files 14, 15: Tables S14 and S15), FMOD had been previously reported to be associated with patient survival. It encodes fibromodulin, an extracellular matrix protein overexpressed in pancreatic ductal adenocarcinoma [149]. In our study, methylation of the $F M O D$ gene body in PAAD was associated with response to the Hsp90 inhibitor 17-AAG (17-allylamino-17-demethoxygeldanamycin; Additional file 15: Table S15). FMOD protein expression had been previously associated with PAAD patient survival in the Queensland Centre for Medical Genomics dataset [149]. In other cancer categories, multiple studies have reported an association of upregulation of $F M O D$ with poor patient survival in TCGA glioblastoma 
patients, and its product has been suggested to have an immunosuppressive role, whereas the silencing of FMOD leads to apoptosis in CCLE [150-152].

In our analysis of the CCLE-GDSC data, methylation of a probe and the gene body of TPO (encoding thyroid peroxidase) was associated with response to the c-Met and NPM-ALK inhibitor PF-2341066 in the PAAD category (Additional files 14, 15: Tables S14 and S15). TPO was previously reported to be among the most mutated genes in PAAD patient tumors in TCGA, suggesting a possible combined influence of epigenetic regulation of this gene and the mutational landscape on treatment response [153].

\section{Discussion}

Using patient-derived cell line genomic and drug response data we identified significant associations of 72 important GMDs with drug response and with DNA methylation based on multiple probes across the epigenome. We were able to confirm many associations in independent datasets using direct validation of comparable associations in the NCI-60 and NCI SCLC cancer cell line panels, and indirect evidence from reports on PAAD patient data. Our results provide a resource for future studies of GMDs which may influence methylation of a particular gene of interest, or analyses to explore direct and indirect associations of GMDs with tumor cell line response for specific therapeutic and pharmacological agents. Expression of multiple GMDs was strongly and significantly correlated with response to a variety of agents, even though the associations in the pancancer data were modest. GMD expression had widespread associations with methylation of genes involved in tumor development and progression and in drug response, suggesting multiple overlapping regulatory influences on the epigenome.

When analyzing indirect GMD effects on drug response (Fig. 2B), we used the threshold of the Spearman correlation coefficient $\rho$, to focus on the strongest significant correlations of GMD expression with methylation of their targets, and on correlations of methylation of the most strongly associated targets with drug response. Individual GMDs also had multiple statistically significant weaker correlations with their targets which we did not report. For example, we identified 1,905 strong significant correlations of GMD expression with methylation of individual probes in the pancancer dataset satisfying $p<1.389 \times 10^{-8}$ and Spearman $|\rho|>0.5$ (Additional file 4: Table S4). When examining weaker GMD-probe correlations using the same significance threshold of $p<1.389 \times 10^{-8}$, we found 24,904 associations with $|\rho|>0.4$, and 254,827 correlations with $|\rho|>0.3$ (data not shown). These results suggest common and complex influence of GMDs on DNA methylation in tumor cells. Weaker associations may indicate important biological influences of GMDs on cancer cell regulation, possibly under specific conditions or in subsets of tumor cells with specific mutational and/or expression profiles.

Many compounds, e.g., MS-275, JQ12, LAQ824, tubastatin A, VNLG/124, AR-42, CUDC-101, belinostat, CAY10603, vorinostat, panobinostat, UNC0638, SGC0946, JQ1, I-BET-762, and PFI-1, included HDAC inhibitors, inhibitors of histone methylation, and bromodomain inhibitors directly targeting epigenetic processes. Many of them directly target GMD products, e.g., HDAC1 is one of the targets of vorinostat, and EHMT1 and EHMT2 are targeted by UNC0638 [71].

While the associations of methylation of the target probes and gene regions with $\log (\mathrm{IC} 50)$ may suggest a possible regulation of drug sensitivity or resistance resulting from DNA methylation on gene expression, many correlations with methylation targets involved epigenetic agents, which may suggest additional epigenetic mechanisms. Examples include the HDACi panobinostat, vorinostat, and AR-42 and the bromodomain inhibitor I-BET-762 (Additional files 12, 13, 14, 15: Tables S12S15). Examples of correlations of methylation of target genes with response to epigenetic drugs include methylation of DAPK3, DDA1, NNMT, MAPK, TREX1, and $A B C C 3$. Even though methylation of those genes was measured prior to treatment, such genes may not necessarily directly affect sensitivity or resistance to epigenetic drugs. While a direct involvement of their products in the response to epigenetic agents is possible, another potential explanation could be that correlations involving methylation of specific target genes may indicate more global influences of different levels of GMD expression on epigenome methylation prior to treatment. In that case, methylation of specific target genes could be a marker of the overall epigenetic activity of one or more GMDs affecting multiple target genes, rather that indicate a direct influence of a specific target gene on drug response. Furthermore, in addition to their effect on DNA methylation, many GMDs analyzed in this study have other epigenetic or regulatory roles which are targeted by some of the agents. Some GMDs, e.g., HDACs, may indirectly regulate gene expression by modifying a diverse set of protein targets including transcription factors [154]. Further biological investigation may be needed to address whether the correlations of drug response with DNA methylation of target genes which were associated with GMD expression may be explained by the mechanisms involving the action of specific target gene products (e.g., by an effect of a transporter on a drug concentration within a cancer cell) or by broad non-specific 
effects of pretreatment GMD expression, which affects DNA methylation of multiple genes in the epigenome.

Our methylation dataset was restricted to the combined measurements of 5-mC and 5-hmC using the Illumina Infinium HumanMethylation450 BeadChip array. The products of several GMDs analyzed in this study, e.g., TET1, TET2, TET3, and TDG, generate 5-fC and 5-caC, whereas MGMT demethylates $\mathrm{O}^{6}$-meG, and the action of ALKBH2, and ALKBH3 results in the removal of $\mathrm{N}^{1}$-meA and $\mathrm{N}^{3}$-meC [1, 13, 37-39] (Additional file 1: Table S1). Drug resistance mechanisms involving some of these pathways, such as the role of MGMT expression in temozolomide resistance, were not detected in our study which used in vitro assay measures, likely because temozolomide is a prodrug which is converted to an active compound in the body, but possibly inconsistently in in vitro screening assays [155]. Similarly, we did not analyze methyladenine modifications as they were not captured in the available methylation data.

Altered GMD function in tumors can arise both from DNA mutations and transcriptional changes $[4,10,144]$. We analyzed the variation in GMD expression and did not examine GMD mutation status. As some GMDs may also have gain-of-function or loss-of-function variants in malignant cells, future large-scale analyses may investigate how drug response of tumor cells may be jointly influenced by DNA and protein sequence changes in GMDs, their copy number variation, gene fusions involving GMDs, and variation in GMD expression. Drug response may also be affected by the sensitivity or resistance mutations acquired by the genes encoding drug targets or by additional genes. Our regression analysis of trametinib response confirmed associations for GADD45A and its putative epigenome targets while accounting for the mutation status of BRAF V600E, KRAS, and NRAS.

Our study provides an extensive reference set of associations between expression of GMDs, their methylation of their epigenome targets, and response to drug treatment in a variety of cancer categories. These results provide a new insight into the epigenetic landscape of molecular interactions in tumors and suggest potential mechanisms of epigenetic influences on tumor cell response to a variety of chemotherapeutic agents.

\section{Conclusions}

We identified multiple associations of GMD expression with drug response and with DNA methylation of individual probes and gene regions in the epigenome. Methylation of many epigenome targets was correlated with response to treatment. Our findings suggest potential direct and indirect influences of GMD expression on drug response, which may be mediated by interconnected regulation of DNA methylation pathways.

\section{Supplementary Information}

The online version contains supplementary material available at https://doi. org/10.1186/s13148-021-01026-4.

Additional file 1: Table S1. Genes directly or indirectly involved in DNA methylation and demethylation which were included in analysis.

Additional file 2: Table S2. Numbers of cell lines with available CCLE and GDSC data, analyzed in each cancer category.

Additional file 3: Table S3. Significant correlations of GMD expression with drug response in the pancancer dataset satisfying $|r|>0.3$ and $p_{\mathrm{FDR}}<0.05$.

Additional file 4: Table S4. Significant strong trans- and cis-correlations of GMD expression with methylation of individual probes in the pancancer dataset satisfying $p<1.389 \times 10^{-8}$ and $|\rho|>0.5$.

Additional file 5: Table S5. Significant strong trans- and cis-correlations of GMD expression with methylation of target gene regions in the pancancer dataset satisfying $p_{\mathrm{FDR}}<0.05$ and $|\rho|>0.5$.

Additional file 6: Table S6. Numbers of cis- and trans-correlations of GMD expression with methylation of individual probes in the pancancer dataset satisfying $p<1.389 \times 10^{-8}$ and $|\rho|>0.5$.

Additional file 7: Table S7. Numbers of cis- and trans-correlations of GMD expression with methylation of gene regions in the pancancer dataset satisfying $p_{\mathrm{FDR}}<0.05$ and $|\rho|>0.5$.

Additional file 8: Table S8. Epigenome-wide Spearman correlations of GMD expression with methylation of individual probes satisfying $p<6.039 \times 10^{-10}$ and $|\rho|>0.5$ in 23 individual cancer categories with at least 10 cell lines.

Additional file 9: Table S9. Epigenome-wide Spearman correlations of GMD expression with methylation of gene regions satisfying $p_{\mathrm{FDR}}<0.05$ and $|\rho|>0.5$ in 23 individual cancer categories with at least 10 cell lines.

Additional file 10: Table S10. Numbers of cis- and trans-correlations of GMD expression with methylation of individual probes in the 23 individual cancer categories with at least 10 cell lines, satisfying $p<6.039 \times 10^{-10}$ and $|\rho|>0.5$.

Additional file 11: Table S11. Numbers of cis- and trans-correlations of GMD expression with methylation of gene regions in the 23 individual cancer categories with at least 10 cell lines, satisfying $p_{\mathrm{FDR}}<0.05$ and $|\rho|$ $>0.5$.

Additional file 12: Table S12. Significant correlations of methylation of target probes with drug response in the pancancer dataset satisfying $p_{\mathrm{FDR}}<0.05$ and a relaxed threshold of $|\rho|>0.4$.

Additional file 13: Table S13. Significant correlations of methylation of target gene regions with drug response in the pancancer dataset satisfying $p_{\text {FDR }}<0.05$ and a relaxed threshold of $|r|>0.4$.

Additional file 14: Table S14. Significant correlations of methylation of target probes with drug response in the 23 individual cancer categories with at least 10 cell lines, satisfying $p_{\mathrm{FDR}}<0.05$ and $|\rho|>0.5$.

Additional file 15: Table S15. Significant correlations of methylation of target gene regions with drug response in the 23 individual cancer categories with at least 10 cell lines, satisfying $p_{\mathrm{FDR}}<0.05$ and $|r|>0.5$.

Additional file 16: Table S16. Significant correlations of GMD expression with drug response in the CCLE-GDSC pancancer dataset from Table 1 which had available data for association analysis in CellminerCDB.

Additional file 17: Table S17. Significant strong trans-correlations of GMD expression with methylation of upstream target gene regions in the 
CCLE-GDSC pancancer dataset from Additional file 5: Table S5 and their associations in the NCl-60 dataset in CellminerCDB.

Additional file 18: Table S18. Significant strong trans-correlations of GMD expression with methylation of upstream target gene regions in the SCLC category of the CCLE-GDSC dataset from Additional file 9: Table S9 and their associations in an independent SCLC dataset of 66 cancer cell lines.

Additional file 19: Data S1. Selected examples of association of GMD expression with DNA methylation of epigenome targets in individual cancer categories.

Additional file 20: Figure S1. Plots showing the distribution of DNA methylation values among 424,840 individual probes, the combined distribution of DNA methylation among 93,591 gene regions, and separate distribution for each gene region category in the 645 cell lines in the pancancer dataset.

Additional file 21: Figure S2. A graphical overview of the numbers of strong trans-correlations between GMD expression and methylation of gene regions in selected cancer categories, satisfying $p_{\mathrm{FDR}}<0.05$ and $|\rho|$ $>0.5$. Numbers of trans- and cis-correlations in these and additional cancer categories are provided in Additional file 11: Table S11. Positive correlations are shown as red bars directed upward, whereas negative correlations are shown as blue bars directed downward. (A) Chronic leukocytic leukemia (CLLE). (B) Colon adenocarcinoma and rectum adenocarcinoma (COAD/READ). (C) Breast cancer (BREAST).

\section{Abbreviations}

5-caC: 5-Carboxylcytosine; 5-fC: 5-Formylcytosine; 2-HG: 2-Hydroxyglutarate; 5-hmC: 5-Hydroxymethylcytosine; 5-hmU: 5-Hydroxymethyluracil; 5-mC: 5-Methylcytosine; 17-AAG: 17-Allylamino-17-demethoxygeldanamycin; 17-DMAG: 17-Dimethylaminoethylamino-17-demethoxygeldanamycin; AICDA (AID): Activation-induced cytidine deaminase; aKG: a-Ketoglutarate; ALL: Acute lymphocytic leukemia; AML (LAML): Acute myeloid leukemia; APOBEC1: Apolipoprotein B mRNA editing activity DNA deaminase 1; APOBEC2: Apolipoprotein B mRNA editing activity DNA deaminase 2; APOBEC3A: Apolipoprotein $B$ mRNA editing activity DNA deaminase 3A; APOBEC3C: Apolipoprotein B mRNA editing activity DNA deaminase 3C; BER: Base excision repair; BLADDER: Bladder cancer; BREAST: Breast cancer; CCLE: Cancer Cell Line Encyclopedia; CESC: Cervical squamous cell carcinoma and endocervical adenocarcinoma; CAMKK2: Calcium/calmodulin-dependent protein kinase kinase 2; CLLE: Chronic lymphocytic leukemia; CDK: Cyclin-dependent kinase; COAD/READ: Colon adenocarcinoma and rectum adenocarcinoma; DA: Duodenal adenocarcinoma; DNMT: DNA methyltransferase; DNMT1: DNA methyltransferase 1; DNMT3A: DNA methyltransferase 3A; DNMT3B: DNA methyltransferase 3B; DNMT3L: DNA methyltransferase 3-like; EC: Esophageal cancer; EGFR: Epidermal growth factor receptor; EZH2: Enhancer of zeste homolog 2; FDR: False discovery rate; GADD45A: Growth arrest and DNA damage 45 protein A; GDSC: Genomics of Drug Sensitivity in Cancer; GEO: Gene Expression Omnibus; GLIOMA: Glioma brain tumors; GMD: Gene affecting DNA methylation or demethylation; H3K9: Histone H3 lysine 9; HDAC: Histone deacetylase; HDACi: Histone deacetylase inhibitors; HNSC: Head and neck squamous cell carcinoma; Hsp90: Heat shock protein 90; IDH: Isocitrate dehydrogenase; IDH1: Isocitrate dehydrogenase 1; IDH2: Isocitrate dehydrogenase 2; IRAK2: Interleukin-1 receptor-associated kinase 2; KIF3: Kinesin superfamily protein 3; LCML: Chronic myelogenous leukemia; LIHC: Liver hepatocellular carcinoma; LIPG: Endothelial lipase; MAP3K14: Mitogen-activated protein kinase kinase kinase 14; MATBCL: Mature B-cell lymphoma; MB: Medulloblastoma; MBD1: Methyl-CpG-binding domain protein 1; MBD2: Methyl-CpG-binding domain protein 2; MBD3: Methyl-CpG-binding domain protein 3; MBD4 (MED1): Methyl-CpG-binding domain protein 4; MEL: Melanoma; MESO: Mesothelioma; MeCP2: Methyl-CpG-binding protein 2; MGMT: O(6)-methylguanine-DNA methyltransferase; MISC: Other miscellaneous categories of cancer including rare cancers or cancers with unspecified information; $\mathrm{MM}$ : Multiple myeloma; $N^{1}$-meA: 1-Methyladenine; $\mathrm{N}^{3}$-meC: 3-Methylcytosine; NCBI GEO: National Center for Biotechnology Information Gene Expression Omnibus; ncRNA: Noncoding RNA; NEURL3: Neutralized E3 ubiquitin protein ligase 3; NFIA: Nuclear factor I; NR1D2: Nuclear receptor subfamily 1 group D member 2; NSCLC: Non-small cell lung cancer; $\mathrm{O}^{6}$-meG: $\mathrm{O}^{6}$-methylguanine; OVARIAN: Ovarian cancer; PAAD: Pancreatic adenocarcinoma; Pancancer: Combined analysis of all cancer categories; PARP: Poly(ADP-ribose) polymerase; PCNA: Proliferating cell nuclear antigen; PNET: Primitive neuroectodermal tumors; PPAP2C: Phosphatidic acid phosphatase type 2C; PRA: Prostate adenocarcinoma; PRC: Polycomb repressive complex; PRC1: Polycomb repressive complex 1; PRC2: Polycomb repressive complex 2; QC: Quality control; RAP1GAP2: RAP1 GTPase activating protein 2; RASEF: The RAS and EF-hand domain containing protein; RCC: Renal cell carcinoma; RIPK: Receptor-interacting protein kinase; SAR: Sarcoma; SCLC: Small cell lung cancer; STAD: Stomach adenocarcinoma; STAT : Signal transducer and activator of transcription; TCGA: The Cancer Genome Atlas; TDG: Thymine-DNA glycosylase; TET: Ten-eleven translocation; TET1: Tet methylcytosine dioxygenase 1 (ten-eleven translocation-1); TET2: Tet methylcytosine dioxygenase 2 (ten-eleven translocation-2); TET3: Tet methylcytosine dioxygenase 3 (ten-eleven translocation-3); THCA: Thyroid carcinoma; TNF: Tumor necrosis factor; UBE2O: (E3-independent) E2 ubiquitin-conjugating enzyme; UCEC: Uterine corpus endometrial carcinoma; USP7 (HAUSP): Herpes virus-associated ubiquitin-specific protease.

\section{Acknowledgements}

We thank the editor, Dr. Tomasz Wojdacz, and two anonymous reviewers for their helpful recommendations which improved the manuscript. We are grateful to Drs. Yingdong Zhao, Lisa McShane, and Dmitriy Sonkin (Biometric Research Program, National Cancer Institute) for helpful suggestions. The views expressed in this article are the personal opinions of the authors and do not necessarily reflect policy of the US National Cancer Institute.

\section{Authors' contributions}

JK conceived and oversaw the study, selected candidate genes, computed average methylation values for gene regions, and drafted the manuscript. SV performed data extraction, processing, and cell line identity matching between the GDSC and CCLE datasets, bioinformatic and statistical association analysis, and participated in the writing of the methodology section. AP developed a supplementary website with multiple search tools for querying the study results and participated in the writing in the methodology section. BAT oversaw the generation and analysis of an independent SCLC methylation, expression, and drug response dataset which was used for the validation of the initial findings, and participated in the validation and interpretation of the study findings. WCR and YP participated in the analysis of SCLC methylation data used for validation, developed the CellminerCDB tool, and participated in the validation of the study findings. JK, SV, YP, and BAT participated in the interpretation of the computational, biological, pharmacological, and clinical study results. All authors edited the manuscript and read and approved its final version.

\section{Funding}

Open Access funding provided by the National Institutes of Health $(\mathrm{NIH})$. The content of this publication does not necessarily reflect the views or policies of the Department of Health and Human Services, nor does mention of trade names, commercial products, or organizations imply endorsement by the U.S. Government. This research was supported [in part] by the Intramural Research Program of the NIH, National Cancer Institute, Center for Cancer Research (Z01 BC 006150). This research was supported [in part] by the Division of Cancer Treatment and Diagnosis of the National Cancer Institute.

\section{Availability of data and materials}

All CCLE, GDSC, and SCLC data used in this project are publicly available online. Information about their location is provided in the Methods section. The significant findings from our study, which are reported in Additional file 1, 3-15: Tables S1 and S2-S15, are available in a searchable format at https://brb. nci.nih.gov/gmdtables/.

\section{Ethics approval and consent to participate}

Not applicable.

\section{Consent for publication}

Not applicable. All authors concur with publication of this manuscript.

\section{Competing interests}

The authors declare that they have no competing interests. 


\section{Author details}

${ }^{1}$ Biometric Research Program, Division of Cancer Treatment and Diagnosis, National Cancer Institute, 9609 Medical Center Dr., Rockville, MD 20850, USA.

${ }^{2}$ General Dynamics Information Technology (GDIT), 3150 Fairview Park Drive, Falls Church, VA 22042, USA. ${ }^{3}$ Developmental Therapeutics Branch, Center for Cancer Research, National Cancer Institute, NIH, Bethesda, MD 20892, USA. ${ }^{4}$ Molecular Pharmacology Program, Division of Cancer Treatment and Diagnosis, National Cancer Institute, Bethesda, MD 20892, USA.

Received: 24 August 2020 Accepted: 10 February 2021

Published online: 06 March 2021

\section{References}

1. Pleyer L, Greil R. Digging deep into "dirty" drugs_-modulation of the methylation machinery. Drug Metab Rev. 2015;47:252-79.

2. Anwar SL, Krech T, Hasemeier B, Schipper E, Schweitzer N, Vogel A, et al. Loss of imprinting and allelic switching at the DLK1-MEG3 locus in human hepatocellular carcinoma. PLoS ONE. 2012;7:e49462.

3. Plass C, Soloway PD. DNA methylation, imprinting and cancer. Eur J Hum Genet. 2002;10:6-16.

4. Shen $\mathrm{H}$, Laird PW. Interplay between the cancer genome and epigenome. Cell. 2013;153:38-55.

5. Wilson AS, Power BE, Molloy PL. DNA hypomethylation and human diseases. Biochim Biophys Acta. 2007;1775:138-62.

6. Cheng Y, He C, Wang M, Ma X, Mo F, Yang S, et al. Targeting epigenetic regulators for cancer therapy: mechanisms and advances in clinical trials. Signal Transduct Target Ther. 2019:4:62

7. Baylin SB. DNA methylation and gene silencing in cancer. Nat Clin Pract Oncol. 2005:2(Suppl 1):S4-11.

8. Herman JG, Merlo A, Mao L, Lapidus RG, Issa JP, Davidson NE, et al. Inactivation of the CDKN2/p16/MTS1 gene is frequently associated with aberrant DNA methylation in all common human cancers. Cancer Res. 1995:55:4525-30.

9. Das PM, Singal R. DNA methylation and cancer. J Clin Oncol. 2004;22:4632-42

10. Plass C, Pfister SM, Lindroth AM, Bogatyrova O, Claus R, Lichter P. Mutations in regulators of the epigenome and their connections to global chromatin patterns in cancer. Nat Rev Genet. 2013;14:765-80.

11. Davalos V, Martinez-Cardus A, Esteller M. The epigenomic revolution in breast cancer: From single-gene to genome-wide next-generation approaches. Am J Pathol. 2017;187:2163-74.

12. Jung H, Kim HS, Kim JY, Sun JM, Ahn JS, Ahn MJ, et al. DNA methylation loss promotes immune evasion of tumours with high mutation and copy number load. Nat Commun. 2019;10:4278.

13. Shen L, Song CX, He C, Zhang Y. Mechanism and function of oxidative reversal of DNA and RNA methylation. Annu Rev Biochem. 2014:83:585-614.

14. Sedgwick B. Repairing DNA-methylation damage. Nat Rev Mol Cell Biol. 2004:5:148-57.

15. Niehrs C, Schafer A. Active DNA demethylation by Gadd45 and DNA repair. Trends Cell Biol. 2012;22:220-7.

16. Siriwardena SU, Chen K, Bhagwat AS. Functions and Malfunctions of Mammalian DNA-Cytosine Deaminases: the known knowns and the known unknowns. Chem Rev. 2016;116:12688-710.

17. Law JA, Jacobsen SE. Establishing, maintaining and modifying DNA methylation patterns in plants and animals. Nat Rev Genet. 2010;11:204-20.

18. Sadakierska-Chudy A, Kostrzewa RM, Filip M. A comprehensive view of the epigenetic landscape part I: DNA methylation, passive and active DNA demethylation pathways and histone variants. Neurotox Res. 2015;27:84-97.

19. Sjolund AB, Senejani AG, Sweasy JB. MBD4 and TDG: multifaceted DNA glycosylases with ever expanding biological roles. Mutat Res. 2013;743-744:12-25.

20. Dominguez PM, Shaknovich R. Epigenetic function of activationinduced cytidine deaminase and its link to lymphomagenesis. Front Immunol. 2014;5:642.
21. Baubec T, Ivanek R, Lienert F, Schubeler D. Methylation-dependent and -independent genomic targeting principles of the MBD protein family. Cell. 2013;153:480-92.

22. Cartron PF, Nadaradjane A, Lepape F, Lalier L, Gardie B, Vallette FM. Identification of TET1 partners that control its DNA-demethylating function. Genes Cancer. 2013;4:235-41.

23. Suetake I, Shinozaki F, Miyagawa J, Takeshima H, Tajima S. DNMT3L stimulates the DNA methylation activity of Dnmt3a and Dnmt3b through a direct interaction. J Biol Chem. 2004;279:27816-23.

24. Cheray M, Pacaud R, Nadaradjane A, Vallette FM, Cartron PF. Specific inhibition of one DNMT1-including complex influences tumor initiation and progression. Clin Epigenetics. 2013;5:9.

25. Bronner C. Control of DNMT1 abundance in epigenetic inheritance by acetylation, ubiquitylation, and the histone code. Sci Signal. 2011;4:pe3.

26. Vidal M. Chromatin modifications by polycomb complexes. Epigenetics in biology and medicine. Boca Raton: CRC Press; 2009. p. 131-54.

27. Denis H, Ndlovu MN, Fuks F. Regulation of mammalian DNA methyltransferases: a route to new mechanisms. EMBO Rep. 2011;12:647-56.

28. Cubenas-Potts C, Matunis MJ. SUMO: a multifaceted modifier of chromatin structure and function. Dev Cell. 2013;24:1-12.

29. Peng L, Yuan Z, Ling H, Fukasawa K, Robertson K, Olashaw N, et al. SIRT1 deacetylates the DNA methyltransferase 1 (DNMT1) protein and alters its activities. Mol Cell Biol. 2011;31:4720-34.

30. Prensner JR, Chinnaiyan AM. Metabolism unhinged: IDH mutations in cancer. Nat Med. 2011:17:291-3.

31. Zhang T, Termanis A, Ozkan B, Bao XX, Culley J, de Lima AF, et al. G9a/ GLP complex maintains imprinted DNA methylation in embryonic stem cells. Cell Rep. 2016;15:77-85.

32. Zhang J, Gao Q, Li P, Liu X, Jia Y, Wu W, et al. S phase-dependent interaction with DNMT1 dictates the role of UHRF1 but not UHRF2 in DNA methylation maintenance. Cell Res. 2011:21:1723-39.

33. Smith ZD, Shi J, Gu H, Donaghey J, Clement K, Cacchiarelli D, et al. Epigenetic restriction of extraembryonic lineages mirrors the somatic transition to cancer. Nature. 2017;549:543-7.

34. Dan J, Rousseau P, Hardikar S, Veland N, Wong J, Autexier C, et al. Zscan4 inhibits maintenance DNA methylation to facilitate telomere elongation in mouse embryonic stem cells. Cell Rep. 2017;20:1936-49.

35. Du J, Johnson LM, Jacobsen SE, Patel DJ. DNA methylation pathways and their crosstalk with histone methylation. Nat Rev Mol Cell Biol. 2015;16:519-32

36. Hagarman JA, Motley MP, Kristjansdottir K, Soloway PD. Coordinate regulation of DNA methylation and $\mathrm{H} 3 \mathrm{~K} 27 \mathrm{me} 3$ in mouse embryonic stem cells. PLoS ONE. 2013;8:e53880

37. Fahrer J, Kaina B. O6-methylguanine-DNA methyltransferase in the defense against $\mathrm{N}$-nitroso compounds and colorectal cancer. Carcinogenesis. 2013;34:2435-42.

38. Zheng G, Fu Y, He C. Nucleic acid oxidation in DNA damage repair and epigenetics. Chem Rev. 2014;114:4602-20

39. Falnes PO, Bjoras $M$, Aas PA, Sundheim O, Seeberg E. Substrate specificities of bacterial and human AlkB proteins. Nucleic Acids Res. 2004:32:3456-61.

40. Moore LD, Le T, Fan G. DNA methylation and its basic function. Neuropsychopharmacology. 2013:38:23-38.

41. Li H, Rauch T, Chen ZX, Szabo PE, Riggs AD, Pfeifer GP. The histone methyltransferase SETDB1 and the DNA methyltransferase DNMT3A interact directly and localize to promoters silenced in cancer cells. J Biol Chem. 2006;281:19489-500

42. Fuks F, Hurd PJ, Deplus R, Kouzarides T. The DNA methyltransferases associate with HP1 and the SUV39H1 histone methyltransferase. Nucleic Acids Res. 2003:31:2305-12.

43. Weinberg DN, Papillon-Cavanagh $S$, Chen H, Yue Y, Chen X, Rajagopalan $\mathrm{KN}$, et al. The histone mark H3K36me2 recruits DNMT3A and shapes the intergenic DNA methylation landscape. Nature. 2019

44. Ulrich CM, Robien K, McLeod HL. Cancer pharmacogenetics: polymorphisms, pathways and beyond. Nat Rev Cancer. 2003;3:912-20.

45. Hagner $\mathrm{N}$, Joerger M. Cancer chemotherapy: targeting folic acid synthesis. Cancer Manag Res. 2010;2:293-301.

46. Jakopovic M, Thomas A, Balasubramaniam S, Schrump D, Giaccone G, Bates SE. Targeting the epigenome in lung cancer: expanding approaches to epigenetic therapy. Front Oncol. 2013:3:261. 
47. Fahy J, Jeltsch A, Arimondo PB. DNA methyltransferase inhibitors in cancer: a chemical and therapeutic patent overview and selected clinical studies. Expert Opin Ther Pat. 2012;22:1427-42.

48. Holbeck SL, Collins JM, Doroshow JH. Analysis of Food and Drug Administration-approved anticancer agents in the NCl60 panel of human tumor cell lines. Mol Cancer Ther. 2010;9:1451-60.

49. Agrawal K, Das V, Vyas P, Hajduch M. Nucleosidic DNA demethylating epigenetic drugs - a comprehensive review from discovery to clinic. Pharmacol Ther. 2018;188:45-79.

50. Fiskus W, Buckley K, Rao R, Mandawat A, Yang Y, Joshi R, et al. Panobinostat treatment depletes EZH2 and DNMT1 levels and enhances decitabine mediated de-repression of JunB and loss of survival of human acute leukemia cells. Cancer Biol Ther. 2009;8:939-50.

51. Li CT, Hsiao YM, Wu TC, Lin YW, Yeh KT, Ko JL. Vorinostat, SAHA, represses telomerase activity via epigenetic regulation of telomerase reverse transcriptase in non-small cell lung cancer cells. J Cell Biochem. 2011;112:3044-53.

52. Lee J, R SH. Cancer epigenetics: mechanisms and crosstalk of a HDAC inhibitor, vorinostat. Chemotherapy (Los Angel). 2013;2:14934.

53. Zhou $X$, Yang $X Y$, Popescu NC. Preclinical evaluation of combined antineoplastic effect of DLC1 tumor suppressor protein and suberoylanilide hydroxamic acid on prostate cancer cells. Biochem Biophys Res Commun. 2012;420:325-30.

54. Arzenani MK, Zade AE, Ming Y, Vijverberg SJ, Zhang Z, Khan Z, et al. Genomic DNA hypomethylation by histone deacetylase inhibition implicates DNMT1 nuclear dynamics. Mol Cell Biol. 2011;31:4119-28.

55. Savickiene J, Treigyte G, Valiuliene G, Stirblyte I, Navakauskiene R. Epigenetic and molecular mechanisms underlying the antileukemic activity of the histone deacetylase inhibitor belinostat in human acute promyelocytic leukemia cells. Anticancer Drugs. 2014;25:938-49.

56. Brown R, Curry E, Magnani L, Wilhelm-Benartzi CS, Borley J. Poised epigenetic states and acquired drug resistance in cancer. Nat Rev Cancer. 2014; 14:747-53.

57. Heyn H, Esteller M. DNA methylation profiling in the clinic: applications and challenges. Nat Rev Genet. 2012;13:679-92.

58. Shen DW, Pouliot LM, Hall MD, Gottesman MM. Cisplatin resistance: a cellular self-defense mechanism resulting from multiple epigenetic and genetic changes. Pharmacol Rev. 2012;64:706-21.

59. Yu W, Jin C, Lou X, Han X, Li L, He Y, et al. Global analysis of DNA methylation by Methyl-Capture sequencing reveals epigenetic control of cisplatin resistance in ovarian cancer cell. PLOS ONE. 2011;6:e29450.

60. Zeller C, Dai W, Steele NL, Siddiq A, Walley AJ, Wilhelm-Benartzi CS, et al. Candidate DNA methylation drivers of acquired cisplatin resistance in ovarian cancer identified by methylome and expression profiling. Oncogene. 2012;31:4567-76.

61. Zhang YW, Zheng Y, Wang JZ, Lu XX, Wang Z, Chen LB, et al. Integrated analysis of DNA methylation and mRNA expression profiling reveals candidate genes associated with cisplatin resistance in non-small cell lung cancer. Epigenetics. 2014;9:896-909.

62. Rosell R, Taron M, Massuti B, Mederos N, Magri I, Santarpia M, et al. Predicting response to chemotherapy with early-stage lung cancer. Cancer J. 2011;17:49-56.

63. Siddik ZH. Cisplatin: mode of cytotoxic action and molecular basis of resistance. Oncogene. 2003;22:7265-79.

64. Flanagan JM, Wilhelm-Benartzi CS, Metcalf M, Kaye SB, Brown R. Association of somatic DNA methylation variability with progressionfree survival and toxicity in ovarian cancer patients. Ann Oncol. 2013:24:2813-8.

65. Yun T, Liu Y, Gao D, Linghu E, Brock MV, Yin D, et al. Methylation of CHFR sensitizes esophageal squamous cell cancer to docetaxel and paclitaxel. Genes Cancer. 2015;6:38-48.

66. Buelow DR, Anderson JT, Pounds SB, Shi L, Lamba JK, Hu S, et al. DNA Methylation-based epigenetic repression of SLC22A4 promotes resistance to cytarabine in acute myeloid leukemia. Clin Transl Sci. 2020.

67. Barretina J, Caponigro G, Stransky N, Venkatesan K, Margolin AA, Kim S, et al. The Cancer Cell Line Encyclopedia enables predictive modelling of anticancer drug sensitivity. Nature. 2012;483:603-7.

68. CCLE Cancer Cell Line Encyclopedia. http://www.broadinstitute.org/ ccle. Accessed 6 Aug 2018.
69. Garnett MJ, Edelman EJ, Heidorn SJ, Greenman CD, Dastur A, Lau KW, et al. Systematic identification of genomic markers of drug sensitivity in cancer cells. Nature. 2012;483:570-5.

70. Genomics of Drug Sensitivity in Cancer. http://www.cancerrxgene.org/. Accessed 5 July 2018.

71. Iorio F, Knijnenburg TA, Vis DJ, Bignell GR, Menden MP, Schubert M, et al. A landscape of pharmacogenomic interactions in cancer. Cell. 2016;166:740-54.

72. Ghandi M, Huang FW, Jane-Valbuena J, Kryukov GV, Lo CC, McDonald ER 3rd, et al. Next-generation characterization of the Cancer Cell Line Encyclopedia. Nature. 2019;569:503-8.

73. Stelzer G, Dalah I, Stein TI, Satanower Y, Rosen N, Nativ N, et al. In-silico human genomics with GeneCards. Hum Genomics. 2011;5(6):709-17.

74. $\mathrm{OMIM}^{\circledR}$ : Online Mendelian Inheritance in Man $^{\circledR}$. An online catalog of human genes and genetic disorders. 2015. http://omim.org. Accessed 27 Feb 2015

75. Genomics of Drug Sensitivity in Cancer data download site. http:// www.cancerrxgene.org/downloads. Accessed 12 June 2018.

76. Bairoch A. The Cellosaurus: a cell line knowledge resource. http://web. expasy.org/cellosaurus/. Accessed 25 April 2017.

77. Stransky N, Ghandi M, Kryukov GV, Garraway LA, Lehár J, Liu M, et al. Pharmacogenomic agreement between two cancer cell line data sets. Nature. 2015;528:84-7.

78. Safikhani Z, Smirnov P, Freeman M, El-Hachem N, She A, Rene Q, et al. Revisiting inconsistency in large pharmacogenomic studies. F1000Res. 2016;5:2333.

79. Haverty PM, Lin E, Tan J, Yu Y, Lam B, Lianoglou S, et al. Reproducible pharmacogenomic profiling of cancer cell line panels. Nature. 2016:533:333-7.

80. CCLE Cancer Cell Line Encyclopedia data download site for RNA-seq RPKM expression data. https://portals.broadinstitute.org/ccle/data,fileC CLE_DepMap_18q3_RNAseq_RPKM_20180718.gct. Accessed 6 Aug 2018.

81. Tsherniak A, Vazquez F, Montgomery PG, Weir BA, Kryukov G, Cowley GS, et al. Defining a cancer dependency map. Cell. 2017;170(564-76):e16.

82. NCBI GEO DNA methylation dataset from the GDSC project. https:// www.ncbi.n/m.nih.gov/geo/query/acc.cgi?acc=GSE68379. Accessed 18 Aug 2018.

83. Zhou W, Laird PW, Shen H. Comprehensive characterization, annotation and innovative use of Infinium DNA methylation BeadChip probes. Nucleic Acids Res. 2017:45:e22.

84. Zhou W, Laird PW, Shen H. Illumina EPIC array methylation hg38 masking annotation. http://zwdzwd.github.io/InfiniumAnnotation. Accessed 17 Sep 2018.

85. Wang D, Yan L, Hu Q, Sucheston LE, Higgins MJ, Ambrosone CB, et al. IMA: an R package for high-throughput analysis of Illumina's 450K Infinium methylation data. Bioinformatics. 2012;28:729-30.

86. Krushkal J, Silvers T, Reinhold WC, Sonkin D, Vural S, Connelly J, et al. Epigenome-wide DNA methylation analysis of small cell lung cancer cell lines suggests potential chemotherapy targets. Clin Epigenetics. 2020;12:93.

87. Illumina Infinium HumanMethylation450K Beadchip Product Files. 2013. https://support.illumina.com/array/array_kits/infinium_humanmethy lation450_beadchip_kit/downloads.html. Accessed 17 Oct 2018.

88. Rakyan VK, Down TA, Balding DJ, Beck S. Epigenome-wide association studies for common human diseases. Nat Rev Genet. 2011;12:529-41.

89. Mishra R, Hanker AB, Garrett JT. Genomic alterations of ERBB receptors in cancer: clinical implications. Oncotarget. 2017:8:114371-92.

90. Kancha RK, von Bubnoff N, Bartosch N, Peschel C, Engh RA, Duyster J. Differential sensitivity of ERBB2 kinase domain mutations towards lapatinib. PLOS ONE. 2011;6:e26760.

91. Shoemaker $\mathrm{RH}$. The NCl60 human tumour cell line anticancer drug screen. Nat Rev Cancer. 2006;6:813-23.

92. CellminerCDB v. 1.2. https://discover.nci.nih.gov/cellminercdb/. Accessed 01 Dec 2020.

93. Luna A, Elloumi F, Varma S, Wang Y, Rajapakse VN, Aladjem MI, et al. CellMiner cross-database (CellMinerCDB) version 1.2: exploration of patient-derived cancer cell line pharmacogenomics. Nucleic Acids Res. 2020. https://doi.org/10.1093/nar/gkaa968. 
94. Rajapakse VN, Luna A, Yamade M, Loman L, Varma S, Sunshine M, et al. CellMinerCDB for integrative cross-database genomics and pharmacogenomics analyses of cancer cell lines. Science. 2018;10:247-64.

95. Reinhold WC, Varma S, Sunshine M, Rajapakse V, Luna A, Kohn KW, et al. The NCl-60 methylome and its integration into CellMiner. Cancer Res. 2017;77:601-12.

96. Polley E, Kunkel M, Evans D, Silvers T, Delosh R, Laudeman J, et al. Small cell lung cancer screen of oncology drugs, investigational agents, and gene and microRNA expression. J Natl Cancer Inst. 2016;108:djw122.

97. NCI Small Cell Lung Cancer Project site. http://sclccelllines.cancer.gov. Accessed 25 Sep 2019.

98. Farcas AM, Blackledge NP, Sudbery I, Long HK, McGouran JF, Rose NR, et al. KDM2B links the polycomb repressive complex 1 (PRC1) to recognition of CpG islands. Elife. 2012;1:e00205.

99. Gan L, Yang Y, Li Q, Feng Y, Liu T, Guo W. Epigenetic regulation of cancer progression by $\mathrm{EZH} 2$ : from biological insights to therapeutic potential. Biomark Res. 2018;6:10.

100. Eckschlager T, PIch J, Stiborova M, Hrabeta J. Histone deacetylase inhibitors as anticancer drugs. Int J Mol Sci. 2017;18.

101. Sashida G, Wang C, Tomioka T, Oshima M, Aoyama K, Kanai A, et al. The loss of Ezh2 drives the pathogenesis of myelofibrosis and sensitizes tumor-initiating cells to bromodomain inhibition. J Exp Med. 2016;213:1459-77.

102. Leal AS, Williams CR, Royce DB, Pioli PA, Sporn MB, Liby KT. Bromodomain inhibitors, JQ1 and I-BET 762, as potential therapies for pancreatic cancer. Cancer Lett. 2017;394:76-87.

103. Andrieu G, Belkina AC, Denis GV. Clinical trials for BET inhibitors run ahead of the science. Drug Discov Today Technol. 2016;19:45-50.

104. Spring L, Bardia A, Modi S. Targeting the cyclin D-cyclin-dependent kinase (CDK) 4/6-retinoblastoma pathway with selective CDK 4/6 inhibitors in hormone receptor-positive breast cancer: rationale, current status, and future directions. Discov Med. 2016;21:65-74.

105. Knudsen ES, Witkiewicz AK. The strange case of CDK4/6 inhibitors: mechanisms, resistance, and combination strategies. Trends Cancer. 2017:3:39-55

106. Gong X, Du J, Parsons SH, Merzoug FF, Webster Y, Iversen PW, et al. Aurora A kinase inhibition is synthetic lethal with loss of the RB1 tumor suppressor gene. Cancer Discov. 2019;9:248-63.

107. Sardina JL, Collombet S, Tian TV, Gomez A, Di Stefano B, Berenguer C, et al. Transcription factors drive Tet2-mediated enhancer demethylation to reprogram cell fate. Cell Stem Cell. 2018;23(727-41):e9.

108. Huang Y, Rao A. Connections between TET proteins and aberrant DNA modification in cancer. Trends Genet. 2014;30:464-74.

109. Mill J, Heijmans BT. From promises to practical strategies in epigenetic epidemiology. Nat Rev Genet. 2013;14:585-94.

110. Huang F, Reeves K, Han X, Fairchild C, Platero S, Wong TW, et al. Identification of candidate molecular markers predicting sensitivity in solid tumors to dasatinib: rationale for patient selection. Cancer Res. 2007:67:2226-38

111. Zhu J, Chen X, Liao Z, He C, Hu X. TGFBI protein high expression predicts poor prognosis in colorectal cancer patients. Int J Clin Exp Pathol. 2015;8:702-10.

112. Ulanovskaya OA, Zuhl AM, Cravatt BF. NNMT promotes epigenetic remodeling in cancer by creating a metabolic methylation sink. Nat Chem Biol. 2013:9:300-6.

113. Shlomi T, Rabinowitz JD. Metabolism: cancer mistunes methylation. Nat Chem Biol. 2013:9:293-4.

114. Poss ZC, Ebmeier CC, Taatjes DJ. The mediator complex and transcription regulation. Crit Rev Biochem Mol Biol. 2013;48:575-608

115. Garvilles RG, Hasegawa T, Kimura H, Sharif J, Muto M, Koseki H, et al. Dual functions of the RFTS domain of Dnmt1 in replication-coupled DNA methylation and in protection of the genome from aberrant methylation. PLOS ONE. 2015;10:e0137509.

116. Miyagawa K, Sakakura C, Nakashima S, Yoshikawa T, Kin S, Nakase Y, et al. Down-regulation of RUNX1, RUNX3 and CBF $\beta$ in hepatocellular carcinomas in an early stage of hepatocarcinogenesis. Anticancer Res. 2006;26:3633-43.

117. Inge TH, Casson LK, Priebe W, Trent JO, Georgeson KE, Miller DM, et al. Importance of Sp1 consensus motifs in the MYCN promoter. Surgery. 2002;132:232-8.
118. Dhuriya YK, Sharma D. Necroptosis: a regulated inflammatory mode of cell death. J Neuroinflammation. 2018;15:199.

119. Liu JY, Zeng QH, Cao PG, Xie D, Chen X, Yang F, et al. RIPK4 promotes bladder urothelial carcinoma cell aggressiveness by upregulating VEGFA through the NF-KB pathway. Br J Cancer. 2018;1 18:1617-27.

120. Canning P, Ruan Q, Schwerd T, Hrdinka M, Maki JL, Saleh D, et al. Inflammatory signaling by NOD-RIPK2 is inhibited by clinically relevant type II kinase inhibitors. Chem Biol. 2015;22:1174-84.

121. Rhyasen GW, Starczynowski DT. IRAK signalling in cancer. Br J Cancer. 2015;112:232-7.

122. Oceandy D, Amanda B, Ashari FY, Faizah Z, Azis MA, Stafford N. The cross-talk between the TNF- $a$ and RASSF-hippo signalling pathways. Int J Mol Sci. 2019;20.

123. Taniguchi K, Karin M. NF-KB, inflammation, immunity and cancer: coming of age. Nat Rev Immunol. 2018;18:309-24.

124. Lieberman HB. Rad9, an evolutionarily conserved gene with multiple functions for preserving genomic integrity. J Cell Biochem. 2006:97:690-7.

125. Sullivan MR, Bernstein KA. RAD-ical new insights into RAD51 regulation. Genes (Basel). 2018:9:629.

126. Yan S, Sorrell M, Berman Z. Functional interplay between ATM/ATRmediated DNA damage response and DNA repair pathways in oxidative stress. Cell Mol Life Sci. 2014;71:3951-67.

127. Shi Z, Gao H, Bai XC, Yu H. Cryo-EM structure of the human cohesinNIPBL-DNA complex. Science. 2020;368:1454-9.

128. LiT, Chen ZJ. The CGAS-cGAMP-STING pathway connects DNA damage to inflammation, senescence, and cancer. J Exp Med. 2018:215:1287-99.

129. Yan N. Immune diseases associated with TREX1 and STING dysfunction. J Interferon Cytokine Res. 2017;37:198-206.

130. Pantelidou C, Sonzogni O, De Oliveria TM, Mehta AK, Kothari A, Wang $D$, et al. PARP inhibitor efficacy depends on CD8(+) T-cell recruitment via intratumoral STING pathway activation in BRCA-deficient models of triple-negative breast cancer. Cancer Discov. 2019;9:722-37.

131. Sen T, Rodriguez BL, Chen L, Corte CMD, Morikawa N, Fujimoto J, et al. Targeting DNA damage response promotes antitumor immunity through STING-mediated T-cell activation in small cell lung cancer. Cancer Discov. 2019;9:646-61.

132. Della Corte CM, Sen T, Gay CM, Ramkumar K, Diao L, Cardnell RJ, et al. STING pathway expression identifies NSCLC with an immune-responsive phenotype. J Thorac Oncol. 2020;15:777-91.

133. Naour JL, Zitvogel L, Galluzzi L, Vacchelli E, Kroemer G. Trial watch: STING agonists in cancer therapy. Oncolmmunology. 2020:9:1777624.

134. Wan DS, Jiang W, Hao JW. Research advances in how the cGAS-STING pathway controls the cellular inflammatory response. Front Immunol. 2020;11.

135. Shu Z, Smith S, Wang L, Rice MC, Kmiec EB. Disruption of muREC2/ RAD51L1 in mice results in early embryonic lethality which can be partially rescued in a p53(-/-) background. Mol Cell Biol. 1999:19:8686-93.

136. Lee JS, Xiao J, Patel P, Schade J, Wang J, Deneen B, et al. A novel tumor-promoting role for nuclear factor IA in glioblastomas is mediated through negative regulation of p53, p21, and PAl1. Neuro Oncol. 2014:16:191-203.

137. Lin M, Li Y, Qin S, Jiao Y, Hua F. Ubiquitin-like modifier-activating enzyme 7 as a marker for the diagnosis and prognosis of breast cancer. Oncol Lett. 2020;19:2773-84

138. Trimpert C, Wesche D, de Groot T, Pimentel Rodriguez MM, Wong V, van den Berg DTM, et al. NDFIP allows NEDD4/NEDD4L-induced AQP2 ubiquitination and degradation. PLoS ONE. 2017;12:e0183774.

139. Jane EP, Premkumar DR, Thambireddy S, Golbourn B, Agnihotri S, Bertrand $\mathrm{KC}$, et al. Targeting NAD(+) biosynthesis overcomes panobinostat and bortezomib-induced malignant glioma resistance. Mol Cancer Res. 2020;18:1004-17.

140. Min DJ, Vural S, Krushkal J. Association of transcriptional levels of folatemediated one-carbon metabolism-related genes in cancer cell lines with drug treatment response. Cancer Genet. 2019;237:19-38.

141. Arnoult N, Correia A, Ma J, Merlo A, Garcia-Gomez S, Maric M, et al. Regulation of DNA repair pathway choice in $S$ and $G 2$ phases by the NHEJ inhibitor CYREN. Nature. 2017;549:548-52.

142. Sugiyama Y, Hatano N, Sueyoshi N, Suetake I, Tajima S, Kinoshita E, et al. The DNA-binding activity of mouse DNA methyltransferase 1 
is regulated by phosphorylation with casein kinase 1delta/epsilon. Biochem J. 2010;427:489-97.

143. Sauvageau M, Sauvageau G. Polycomb group proteins: multifaceted regulators of somatic stem cells and cancer. Cell Stem Cell. 2010;7:299-313.

144. Poirier JT, Gardner EE, Connis N, Moreira AL, de Stanchina E, Hann CL, et al. DNA methylation in small cell lung cancer defines distinct disease subtypes and correlates with high expression of EZH2. Oncogene. 2015:34:5869-78.

145. Gardner EE, Lok BH, Schneeberger VE, Desmeules P, Miles LA, Arnold PK, et al. Chemosensitive relapse in small cell lung cancer proceeds through an EZH2-SLFN11 axis. Cancer Cell. 2017;31:286-99.

146. Sen T, Gay CM, Byers LA. Targeting DNA damage repair in small cell lung cancer and the biomarker landscape. Transl Lung Cancer Res. 2018;7:50-68

147. Horie M, Saito A, Ohshima M, Suzuki HI, Nagase T. YAP and TAZ modulate cell phenotype in a subset of small cell lung cancer. Cancer Sci. 2016;107:1755-66.

148. Tlemsani C, Pongor L, Elloumi F, Girard L, Huffman KE, Roper N, et al. SCLC-CellMiner: a resource for small cell lung cancer cell line genomics and pharmacology based on genomic signatures. Cell Rep. 2020;33:108296.

149. Tian C, Clauser KR, Ohlund D, Rickelt S, Huang Y, Gupta M, et al. Proteomic analyses of ECM during pancreatic ductal adenocarcinoma progression reveal different contributions by tumor and stromal cells. Proc Natl Acad Sci U S A. 2019;116:19609-18.
150. Han J, Puri RK. Analysis of the cancer genome atlas (TCGA) database identifies an inverse relationship between interleukin-13 receptor a1 and $a 2$ gene expression and poor prognosis and drug resistance in subjects with glioblastoma multiforme. J Neurooncol. 2018;136:463-74.

151. Xiong J, Bing Z, Su Y, Deng D, Peng X. An integrated mRNA and microRNA expression signature for glioblastoma multiforme prognosis. PLOS ONE. 2014;9:e98419.

152. Choudhury A, Derkow K, Daneshmanesh AH, Mikaelsson E, Kiaii S, Kokhaei $P$, et al. Silencing of ROR1 and FMOD with siRNA results in apoptosis of CLL cells. Br J Haematol. 2010;151:327-35.

153. Cui NP, Zhang JS, Huang HT, Lu LG. Epigenetic regulator ARID1A and stem cell transcription factor SOX9 in the maintenance of pancreatic ductal cell differentiation state and development of intraductal papillary mucinous neoplasia (IPMN) and pancreatic ductal adenocarcinoma (PDAC). Transl Cancer Res. 2018;7:S748-51.

154. Kim HJ, Bae SC. Histone deacetylase inhibitors: molecular mechanisms of action and clinical trials as anti-cancer drugs. Am J Transl Res. 2011;3:166-79.

155. Lee SY. Temozolomide resistance in glioblastoma multiforme. Genes Dis. 2016:3:198-210.

\section{Publisher's Note}

Springer Nature remains neutral with regard to jurisdictional claims in published maps and institutional affiliations.
Ready to submit your research? Choose BMC and benefit from:

- fast, convenient online submission

- thorough peer review by experienced researchers in your field

- rapid publication on acceptance

- support for research data, including large and complex data types

- gold Open Access which fosters wider collaboration and increased citations

- maximum visibility for your research: over 100M website views per year

At BMC, research is always in progress.

Learn more biomedcentral.com/submissions 